\title{
Targeting Iron Acquisition Blocks Infection with the Fungal Pathogens Aspergillus fumigatus and Fusarium oxysporum
}

\author{
Sixto M. Leal, Jr. ${ }^{1,2}$, Sanhita Roy ${ }^{1}$, Chairut Vareechon ${ }^{1,2}$, Steven deJesus Carrion ${ }^{1,2}$, Heather Clark ${ }^{1,2}$, \\ Manuel S. Lopez-Berges ${ }^{3}$, Antonio diPietro ${ }^{3}$, Marcus Schrettl ${ }^{4}$, Nicola Beckmann ${ }^{4}$, Bernhard RedI ${ }^{4}$, \\ Hubertus Haas ${ }^{4}$, Eric Pearlman ${ }^{1,2 *}$
}

1 Department of Ophthalmology and Visual Sciences, Case Western Reserve University, Cleveland, Ohio, United States of America, 2 Department of Pathology, Case Western Reserve University, Cleveland, Ohio, United States of America, 3 Department of Genetics, Universidad de Cordoba, Cordoba, Spain, 4 Department of Molecular Biology, Medical University of Innsbruck, Innsbruck, Austria

\begin{abstract}
Filamentous fungi are an important cause of pulmonary and systemic morbidity and mortality, and also cause corneal blindness and visual impairment worldwide. Utilizing in vitro neutrophil killing assays and a model of fungal infection of the cornea, we demonstrated that Dectin-1 dependent IL-6 production regulates expression of iron chelators, heme and siderophore binding proteins and hepcidin in infected mice. In addition, we show that human neutrophils synthesize lipocalin-1, which sequesters fungal siderophores, and that topical lipocalin-1 or lactoferrin restricts fungal growth in vivo. Conversely, we show that exogenous iron or the xenosiderophore deferroxamine enhances fungal growth in infected mice. By examining mutant Aspergillus and Fusarium strains, we found that fungal transcriptional responses to low iron levels and extracellular siderophores are essential for fungal growth during infection. Further, we showed that targeting fungal iron acquisition or siderophore biosynthesis by topical application of iron chelators or statins reduces fungal growth in the cornea by $60 \%$ and that dual therapy with the iron chelator deferiprone and statins further restricts fungal growth by $75 \%$. Together, these studies identify specific host iron-chelating and fungal iron-acquisition mediators that regulate fungal growth, and demonstrate that therapeutic inhibition of fungal iron acquisition can be utilized to treat topical fungal infections.
\end{abstract}

Citation: Leal SM Jr, Roy S, Vareechon C, Carrion SdJ, Clark H, et al. (2013) Targeting Iron Acquisition Blocks Infection with the Fungal Pathogens Aspergillus fumigatus and Fusarium oxysporum. PLoS Pathog 9(7): e1003436. doi:10.1371/journal.ppat.1003436

Editor: Marta Feldmesser, Albert Einstein College of Medicine, United States of America

Received November 17, 2012; Accepted May 2, 2013; Published July 11, 2013

Copyright: ( $\odot 2013$ Leal et al. This is an open-access article distributed under the terms of the Creative Commons Attribution License, which permits unrestricted use, distribution, and reproduction in any medium, provided the original author and source are credited.

Funding: This work was supported by NIH grants F31 EY019841 (SML), RO1 EY018612 (EP), P30 EY011373 (EP). These studies were also supported by a Research to Prevent Blindness Medical Student Fellowship (SML), unrestricted grants from the Research to Prevent Blindness Foundation (EP), and the Ohio Lions Eye Research Foundation (EP). HH's laboratory was supported by the Austrian Science Foundation grant FWFI282-B09 ERA-NET PathoGenoMics project TRANSPAT. The funders had no role in study design, data collection and analysis, decision to publish, or preparation of the manuscript.

Competing Interests: The authors have declared that no competing interests exist.

*E-mail: Eric.Pearlman@case.edu

\section{Introduction}

Aspergillus and Fusarium are filamentous fungi that cause lethal infections in immune suppressed individuals [1,2]. Additionally, they infect the corneas of immunocompetent individuals, and are a major cause of blindness associated with ocular trauma [3,4]. Although less common, Curoularia, Alternaria, and Penicillium species also cause keratitis [3]. Globally, the world health organization estimates that 1.8 million people in developing nations are blinded annually from corneal ulcers; furthermore, in developing nations, up to $65 \%$ of total corneal ulcers are caused by fungal infection, with approximately 1 million cases occurring annually in Asia and Africa [5-7].

Treatment regimens for fungal keratitis are often ineffective, with up to $60 \%$ of fungal keratitis cases requiring corneal transplantation [3]. Given the limited treatment options, there is a pressing need to develop new treatment strategies. In this effort, we recently demonstrated that inhibitors of fungal anti-oxidative responses enhanced fungal clearance in vivo and improved disease outcome [8]. As iron is essential for the redox reactions of major fungal antioxidants, including thioredoxin-dependent peroxiredoxases [9,10], and fungal iron acquisition mutants are more susceptible to oxidative stress [11], we hypothesized that targeting fungal iron acquisition may represent a potential new avenue for treatment of fungal infections.

Fungal iron acquisition primarily involves the production of hydroxamate-type siderophores that are secreted into the environment, bind iron with high affinity, and are then captured by specific siderophore receptors on the fungal cell membrane [12]. Specifically, the A. fumigatus siderophore biosynthesis pathway originates with the sidA gene, which encodes ornithine$\mathcal{N}^{5}$-oxygenase, resulting in conversion of ornithine to $\mathcal{N}^{5}$-hydroxyornithine [13]. Utilizing this essential precursor, the siderophore biosynthesis pathway leads to either intracellular or extracellular siderophores. The sid $C$ gene product is required for production of the intracellular siderophores, ferricrocin $(\mathrm{FC})$ and hydroxyferricrocin (HFC), whereas the sidF and sidD gene products are required for production of the extracellular siderophores, fusar- 


\section{Author Summary}

Fungal pathogens, in addition to causing life-threatening systemic disease, can also invade the cornea and cause blindness and visual impairment. In the current study, we examined the role of iron acquisition in corneal infections caused by Aspergillus and Fusarium. We first demonstrated that expression of iron chelators, heme, siderophore binding proteins and hepcidin is elevated in infected corneas. Secondly, we showed impaired in vivo growth in Aspergillus and Fusarium with mutations in the pathway leading to production of iron-binding siderophores. These mutants were also more susceptible to killing by human neutrophils. Based on these observations, we targeted these pathways using topical iron chelators and found that they blocked fungal growth in the cornea. Finally, as statins target the enzyme HMG-CoA reductase, which is required for siderophore and ergosterol biosysnthesis, we found that topical statins inhibited fungal growth and reduced infection, and showed that combined treatment with the iron chelator deferiprone and statins had an additive effect on fungal infection. Together, these studies demonstrate that therapeutic inhibition of fungal iron acquisition can be utilized to treat topical fungal infections.

inine $\mathrm{G}$ (FusC) and triacetylfusarinine $\mathrm{C}$ (TAFG) [13]. The sidG gene product is required to generate TAFC from FusC [13], whereas both the $s i d H$ and sidI gene products are required to incorporate mevalonate into the structure of extracellular siderophores [14]. As mevalonate biosynthesis is dependent on HMGCoA reductase and this enzyme is inhibited by statins, we hypothesized that statin-mediated inhibition of fungal HMG-CoA reductase may restrict fungal iron acquisition in vivo.

Fungal siderophores are secreted into mammalian tissues during infection where they compete with host iron sequestration defenses. Under homeostatic conditions, free iron is maintained at relatively low levels by iron-binding proteins such as transferrin and ferritin [15]. In addition, mucosal secretions contain high concentrations of lactoferrin, which binds iron, and lipocalin-1, which sequesters fungal siderophores [16,17]. However, tissue damage during infection increases extracellular iron levels by releasing intracellular labile iron, ferritin, and heme-containing proteins [18]. Infection also stimulates both local and systemic immune defenses to counter microbial iron acquisition. Resident cells can secrete iron-sequestering proteins and chemotactic cytokines, which recruit neutrophils to the site of infection. Neutrophils also release pre-formed and de novo synthesized iron sequestering proteins such as lactoferrin, Lcn-2, and the hemoglobin binding protein haptoglobin [19]. Furthermore, neutrophilmediated oxidation is likely to increase the microbial requirement for iron to fuel iron-dependent anti-oxidative defenses. Lastly, production of cytokines such as IL-6 and IL-22 can induce local and systemic synthesis of the peptide hormone hepcidin, which degrades the iron exporter ferroportin and traps iron inside host cells [20-22].

In the current study, we examined the role of host iron sequestration and fungal iron acquisition in a murine model of Aspergillus and Fusarium corneal infection. We show that Dectin-1 and IL-6 regulate expression of genes involved in iron sequestration and that fungal growth positively correlates with serum iron levels. Using mutant $A$. fumigatus and $F$. oxysporum strains, we also demonstrate that fungal transcriptional responses to low iron levels and mevalonic acid-dependent extracellular siderophore biosyn- thesis, but not intracellular siderophores or reductive iron assimilation, are essential for fungal growth in vitro and during infection. Lastly, using iron chelators, siderophore binding proteins, and siderophore biosynthesis inhibitors including statins we provide proof-of-concept that targeting fungal iron acquisition enhances fungal clearance from infected tissues and may represent a new avenue for treatment of fungal infections.

\section{Results}

\section{Dectin- 1 and IL-6 regulate local and systemic endogenous host iron-sequestration pathways}

As we previously showed that Dectin-1 mediates neutrophil recruitment into the cornea during Aspergillus infection [23], and IL-6 is known to mediate hepatic hepcidin production [15], we examined the role of Dectin-1 and IL-6 in iron-sequestration during fungal infection. To determine if fungal infection of the cornea initiates an iron-sequestration response, we infected C57BL/6, Dectin- $1^{-/-}$, and IL- $6^{-/-}$mice intrastromally with A. fumigatus dsRed conidia as described [8]. After $10 \mathrm{~h}$, corneas were dissected and homogenized, and IL-6 was quantified by ELISA. Figure 1A shows increased IL-6 in the corneas of infected compared with naive C57BL/6 mice at $10 \mathrm{~h}$ post-infection; however, IL-6 was not significantly elevated in Dectin-1 ${ }^{-1-}$ corneas. Serum IL-6 was undetectable in naïve C57BL/6 mice; however, at $24 \mathrm{~h}$ post-infection serum IL-6 increased to $400 \rho \mathrm{g} / \mathrm{ml}$ in infected C57BL/6 mice (Figure 1B). As hepcidin expression is induced by IL-6 [15], we examined hepcidin expression in livers of infected $\mathrm{C} 57 \mathrm{BL} / 6$ and IL- $6^{-/-}$mice at $24 \mathrm{~h}$ post-infection. Figure 1C shows that hepcidin expression was elevated 10 -fold in infected compared with naïve C57BL/6 mice, whereas expression was significantly lower in infected IL- $6^{-/-}$mice.

During fungal infection of humans and mice, neutrophils comprise $>95 \%$ of the cellular infiltrate in the cornea and are likely the predominant source of gene transcripts [23,24]. Therefore, prior to examining de novo transcription of ironsequestering genes during infection we first quantified the number of neutrophils infiltrating C57BL/ 6 and IL- $6^{-/-}$infected corneas by flow cytometry using the Ly6G NIMP-R14 monoclonal antibody. As shown in Figure 1D, there was no significant difference in neutrophil numbers between infected C57BL/ 6 and IL- $6^{-1-}$ mice. RNA was then isolated from C57BL/6 and IL- $6^{-/-}$corneas at $24 \mathrm{~h}$ post-infection, and gene expression was measured by Q-PCR.

Compared with naïve C57BL/6 corneas, expression of the iron chelating protein transferrin (TF) was up-regulated 10-fold, transferrin receptor 2 (TR2) expression was up-regulated 400fold, and lactoferrin receptor/intelectin-1 (LR) was elevated 3-fold in infected corneas (Figure 1E). In contrast, these genes were not elevated in infected IL- $6^{-/-}$corneas. Lactoferrin and transferrin receptor 1 expression were not elevated in C57BL/ 6 corneas, most likely due to these proteins being pre-formed in neutrophils [19].

During infection, lysed cells release heme, which microbes can utilize as a source of iron [18]. To restrict microbial access to heme, mammals produce hemopexin $(\mathrm{Hpx})$ that binds to heme, and haptoglobin $(\mathrm{HptG})$, which binds hemoglobin [15]. Figure 1E also shows that expression of Hpx and HptG in infected C57BL/6 mice is up-regulated 200 -fold compared with naïve mice, whereas expression is $<10$-fold increased in infected IL- $6^{-/-}$mice.

In a low iron environment, microbial siderophores bind iron with high affinity and are subsequently internalized through siderophore transporters on the fungal cell membrane [12,25]. Humans encode lipocalin-1 (Lcn-1) and lipocalin-2 (Lcn-2), whereas mice only express Lcn-2 [26]. Both Lcn-1 and Lcn-2 
A

Cornea IL-6

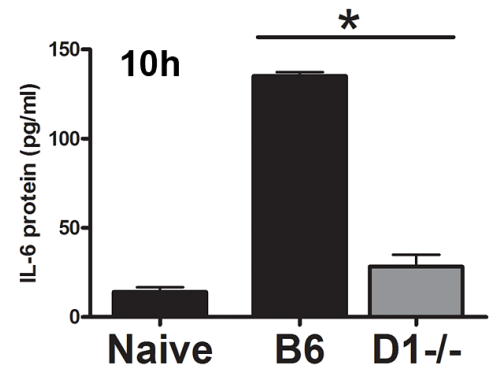

D

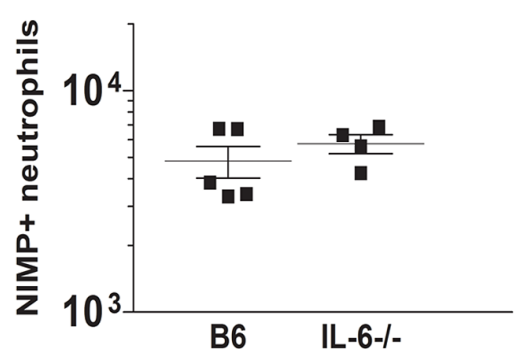

F Neutrophil Len-1

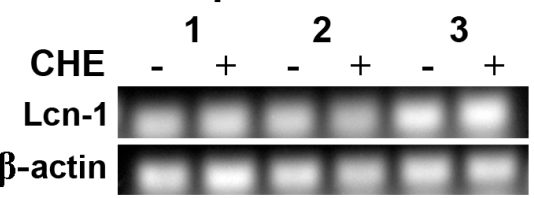

B

Serum IL-6

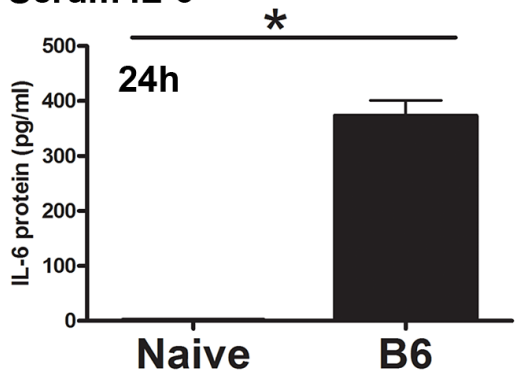

E

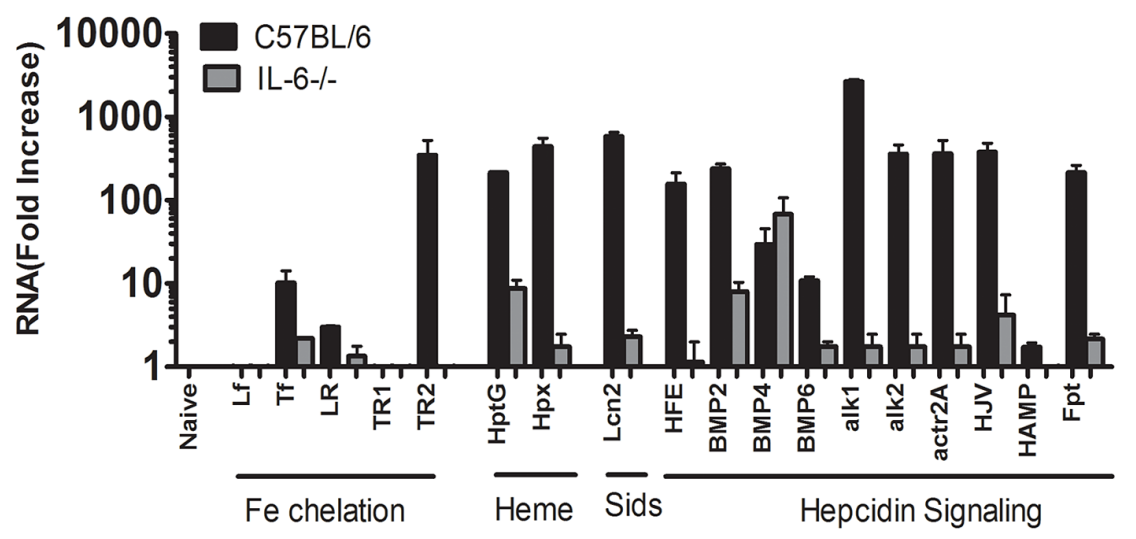

Figure 1. Expression of local and systemic iron-sequestration proteins in Aspergillus fumigatus infected corneas. A. IL-6 production in corneas of C57BL/6 and Dectin- $1^{-1-}$ mice $10 \mathrm{~h}$ after infection with A. fumigatus. B. Serum IL-6 at $24 \mathrm{~h}$ post-infection was quantified by ELISA C. Total liver hepcidin gene expression was quantified using qPCR $24 \mathrm{~h}$ after corneal infection. D. Total neutrophil numbers in corneas of C57BL/6 and IL- $6^{-1-}$ mice $24 \mathrm{~h}$ post-infection. Neutrophils were incubated with the Ly6G NIMP-R14 Ab, and examined by flow cytometry. (Data points represent individual corneas) E. RNA was extracted from corneas of infected mice $24 \mathrm{~h}$ post-infection, and genes encoding proteins involved in iron chelation, heme or siderophore sequestration, and hepcidin signaling were examined by qPCR. F. Lcn-1 gene expression in peripheral blood neutrophils from healthy volunteers after $2 \mathrm{~h}$ incubation with crude hyphal extract (CHE). (Data in panels A, B, C and E are mean +/- SD of 5 mice per group; Data in panel $\mathrm{F}$ are from three separate human donors). Abbreviations: B6-C57BL/6, CHE- crude Aspergillus hyphal extract, D1-Dectin-1, Lf-lactoferrin, Tftransferrin, LR- lactoferrin receptor, TR- transferrin receptor, HptG- haptoglobin, Hpx- hemopexin, Lcn-lipocalin, HFE- human hemochromatosis protein, BMP-bone morphogenetic protein, Alk-activin receptor-like kinase, Actr-actin-related protein, HJV- hemojuvelin, HAMP-hepcidin, Fptferroportin.

doi:10.1371/journal.ppat.1003436.g001

bind to bacterial siderophores, but only Lcn-1 binds fungal hydroxamate-type siderophores $[17,26]$. We found that in infected C57BL/6 corneas, Lcn-2 expression is elevated 600-fold compared with 2-fold in IL-6 ${ }^{-/-}$mice (Figure 1E).

Although hepcidin is produced systemically by the liver, it is also produced by neutrophils and monocytes after stimulation with IL6 , or by iron-bound transferrin-mediated activation of the transferrin receptor 2/human hemochromatosis protein (HFE) complex [15]. Further, hepcidin induction is increased synergistically by bone-morphogenetic protein (BMP)-mediated activation and signaling through a hemojuvelin/BMP receptor complex [15]. Figure 1E shows that many of the proteins involved in noninflammatory induction of hepcidin are up-regulated at $24 \mathrm{~h}$ postinfection, including HFE protein (200-fold), BMP2 (200-fold), BMP-4 (30-fold), BMP-6 (10-fold), the BMP receptors: alk1 (2000fold) alk2 (300-fold), actr2A (300-fold), and hemojuvelin (300-fold). Interestingly, local hepcidin transcript is only 2-fold up-regulated at $24 \mathrm{~h}$ post-infection. In addition, ferroportin transcripts are upregulated 250-fold during infection (Figure 1E), which may be a secondary response following hepcidin-mediated ferroportin degradation. Figure 1E shows that the transcription of all the hepcidin genes analyzed, except BMP-4, were significantly lower in infected IL- $6^{-/-}$mice compared to infected C57BL/6 mice.

Lcn-1 binds to many hydrophobic molecules including phospholipids at the air-fluid interface in tears [27]. However, Lcn-1 also binds to fungal hydroxamate-type siderophores [17]. Given that human neutrophils store the bacterial-siderophore binding protein Lcn-2 in secondary granules [19], we examined if human neutrophils also produce Lcn-1. Peripheral blood neutrophils ( $>95 \%$ purity) from three healthy human volunteers were incubated for $1 \mathrm{~h}$ in RPMI media in the presence or absence of $A$. fumigatus crude hyphal extract, and Lcn-1 expression was examined by Q-PCR. As shown in Figure 1F, Lcn-1 gene expression was detected in human neutrophils in the presence or absence of Aspergillus hyphal extract, indicating constitutive RNA expression. Taken together, results from this set of studies indicate that following fungal infection of the cornea, Dectin-1 dependent IL-6 production induces local and systemic host responses that limit microbial access to iron. 
Iron availability regulates hyphal growth and the severity of $A$. fumigatus infection

Given that fungal infection initiates an iron sequestration response, we next examined if iron availability regulates fungal growth during infection. Mice were injected intraperitoneally with $5 \mathrm{mg}$ Fe-dextran (90 $\mu$ moles iron) or deferroxamine ( $5 \mathrm{mg}$ ), which is an iron-chelating xenosiderophore that is utilized by $A$. fumigatus [28]. Twenty four hours after the last injection, RFP-expressing $A$. fumigatus (Af-dsRed) conidia were injected into the corneal stroma of C57BL/6 mice [8,23]. After $24 \mathrm{~h}$, total serum iron was quantified in treatment and control groups.

Figure 2A shows that serum iron levels in infected mice were reduced 2-fold compared with naïve mice, indicating systemic iron-sequestration during inflammation [15]. In contrast, mice given systemic Fe-dextran, but not deferroxamine had significantly elevated serum iron compared to vehicle-treated mice. Despite the difference in serum iron levels, Figures 2B, $\mathbf{C}$ show that whereas fungal mass (dsRed) increases over $48 \mathrm{~h}$ in vehicle-treated mice, this was significantly higher in Fe-dextran and deferroxaminetreated mice. Consistent with these data, Figure 2D shows that at $48 \mathrm{~h}$ post-infection, CFU were significantly higher in Fe-dextran and deferroxamine-treated mice compared with control, vehicletreated mice. As filamentous fungi grow by hyphal extension and not cell division, the dsRed measure of fungal mass increases over time, whereas CFU decreases from the initial inoculum [15]. Figures 2B, E, and $\mathbf{F}$ show that corneal opacification was also increased in Fe-dextran and deferroxamine-treated mice compared to vehicle-treated mice, consistent with increased fungal growth. The increased fungal growth in deferroxamine treated mice is likely due to its xenosiderophore function, which can be used by Aspergillus for iron acquisition [28].

To examine the effect of limiting iron availability during infection, C57BL/ 6 corneas were infected with A. fumigatus dsRed conidia as described (12), and the iron chelating protein lactoferrin was added topically $(10.4 \mu \mathrm{g}$ in $8 \mu \mathrm{l})$ at $0 \mathrm{~h}$ and $6 \mathrm{~h}$ post-infection. As shown in Figures 2G-I, infected corneas given topical lactoferrin had significantly less fungal mass (dsRed) and CFU per eye at $24 \mathrm{~h}$ post-infection compared with those given vehicle alone. Taken together with results from Fe-dextran and deferroxamine-treated mice, these studies demonstrate that fungal growth in the cornea is dependent on increased free iron or bio-available iron.

\section{Siderophores and detection of low iron concentrations but not reductive iron assimilation is required for fungal growth during infection}

Given that fungal growth during infection is enhanced by the exogenous xenosiderophore deferroxamine, we next examined the role of endogenous fungal siderophores using A. fumigatus, $F$. oxysporum, and Alternaria brassicicola iron acquisition mutants. The $A$. fumigatus $\Delta$ sidA mutant does not synthesize extracellular or intracellular siderophores [29], whereas the $\Delta$ hapX strain lacks the transcription factor HapX that is activated by low iron concentrations, and which regulates expression of genes involved in iron acquisition including siderophores and repression of irondependent pathways [30].

Figure 3A shows no significant difference in fungal growth in media alone between the parent (WT) strain and the $\Delta$ sidA and $\Delta h a p X$ mutants, indicating that there is no effect of these mutations on fungal growth in the presence of an exogenous source of iron. We next examined the growth of the WT strain and the $\Delta$ sidA and $\Delta$ hap $X$ mutants in the presence of neutrophils, which we recently showed kill hyphae by producing reactive oxygen species [8].
Conidia (spores; 12,500) were cultured $4-6 \mathrm{~h}$ in SDB media to allow germination and production of hyphae and incubated with $1 \times 10^{5}$ neutrophils. In contrast to RPMI media alone in which all strains grew equally, in the presence of human neutrophils, growth of the $\Delta$ sidA and $\Delta h a p X$ mutants was significantly less than the WT parental strain (Figure 3A), indicating that adaptation to iron starvation and siderophores, which requires SidA and HapX is essential for survival in the presence of neutrophils.

Consistent with a role for these genes in virulence, we found that at $48 \mathrm{~h}$ post-infection, corneas infected with the $A$. fumigatus mutant strains $\Delta$ sidA or $\Delta h a p X$ had significantly lower CFU compared with the parent strain (Figure 3B). Figure $\mathbf{S 1}$ shows that corneas infected with the complemented $\Delta$ sidA strain $\operatorname{sid} A^{\mathbf{R}}$ or the complemented $\Delta h a p X$ strain hap $\mathrm{X}^{\mathbf{R}}$, show no significant difference in opacification or CFU as the WT parental strain. In contrast, although the $\Delta f t r A$ mutant lacks a membrane-bound iron transport channel protein and is therefore deficient in cellular uptake of environmental iron [29], there was no significant difference in CFU in this mutant, indicating that this transporter protein is not essential for fungal growth in vivo. Similarly, Figures $3 \mathbf{C}, \mathbf{E}$, and $\mathbf{F}$ show that mice infected with the $\Delta$ sidA or $\Delta h a p X$ mutants have significantly less corneal opacification than the parent $A$. fumigatus strain, whereas the $\Delta f t r A$ mutant was not significantly different. Histological analysis shows a pronounced cellular infiltrate and fungal hyphae in the corneas of mice infected with the parent strain, whereas no hyphae were detected in $\Delta$ sidA infected corneas, indicating that these mutants did not germinate in the cornea (Figure 3D). As with $A$. fumigatus, mice infected with the $F$. oxysporum $\Delta$ hap $X$ strain also exhibit significantly lower CFU than the parent strain at $48 \mathrm{~h}$ post-infection (Figure 3G), and $A$. brassicicola siderophore mutants have significantly less CFU than the parent strain (Figure S2). Figure 3H-J demonstrate that mice infected with the $F$. oxysporum $\Delta h a p X$ strain also exhibit significantly less corneal opacification compared to the parental strain. Figure $\mathbf{S} 1$ shows that corneas infected with the Fusarium complemented $\Delta$ hap $X$ strain hapX $\mathrm{X}^{\mathbf{R}}$ show no significant phenotypic differences from the WT parental strain. Taken together, these data indicate that siderophores have a critical role in fungal growth during infection, whereas reductive iron assimilation is not essential.

\section{Extracellular siderophores are required for fungal infection}

To determine the relative contribution of intracellular versus extracellular siderophores in fungal keratitis, corneas were infected with the $\Delta s i d F$ and $\Delta s i d C$ mutants. Figure $4 B$ shows that GFU from corneas infected with the $\Delta$ sidF mutant that leads to extracellular siderophore production was significantly lower than those infected with WT A. fumigatus, having a similar CFU as $\Delta$ sidA mutants. In contrast, $\Delta$ sidC mutants that regulate production of intracellular siderophores were not significantly different from the parent strain. Consistent with this finding, corneas infected with $\Delta s i d F$ had significantly lower cornea opacity area and intensity values compared with mice infected with WT A. fumigatus, whereas $\Delta$ sidC mutants were not significantly different (Figure $\mathbf{4 C}-\mathbf{E}$ ). Together, these findings indicate that extracellular, but not intracellular siderophores are essential for fungal growth in the cornea and development of keratitis.

To determine the relative contribution of the extracellular siderophores FusC and TAFG, we infected corneas with $\Delta$ sidD mutants, which do not produce extracellular siderophores, or with $\Delta$ sidG mutants, which produce FusC but not TAFC, and compared them with the parent $A$. fumigatus strain that produces both FusC and TAFC [13]. Figure 4B shows that mice infected 
A

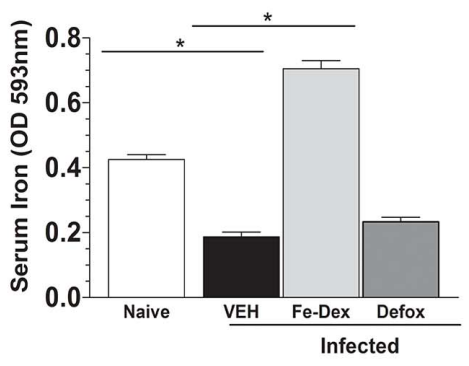

D

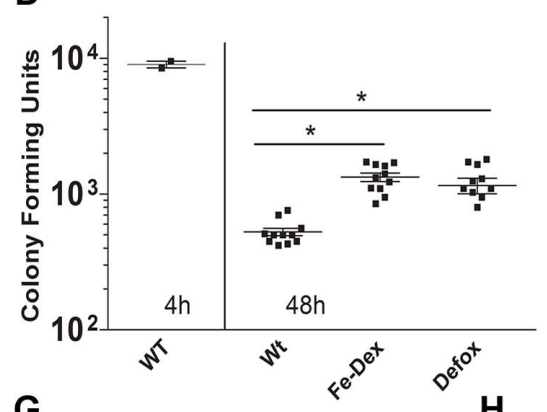

G

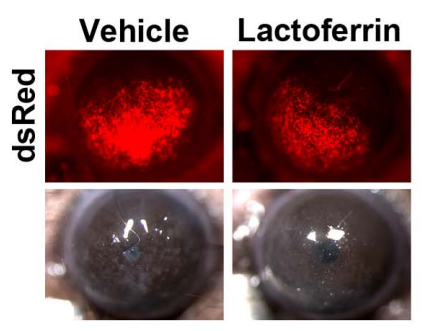

B

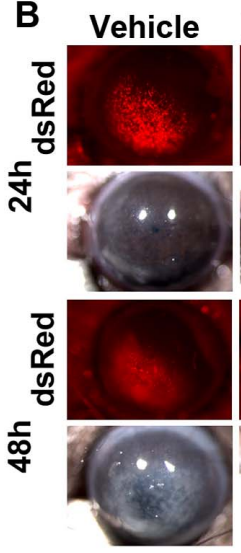

E

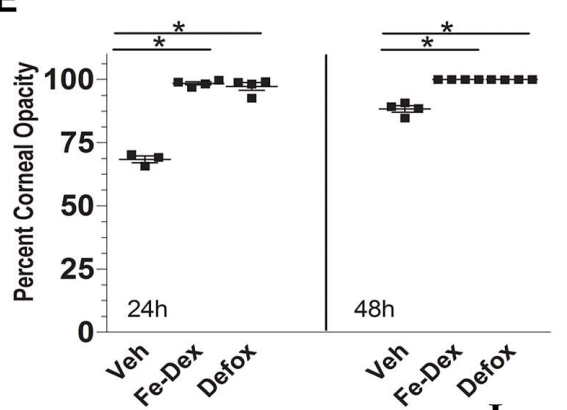

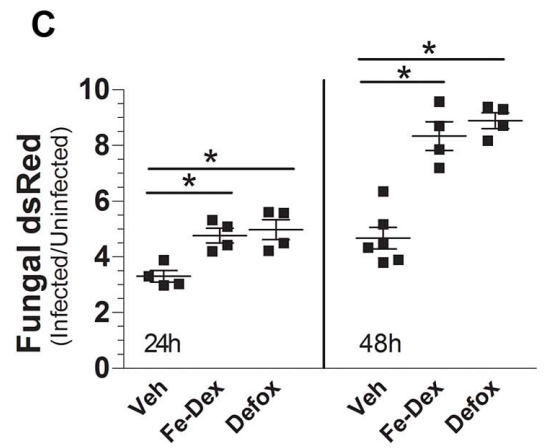

$F$

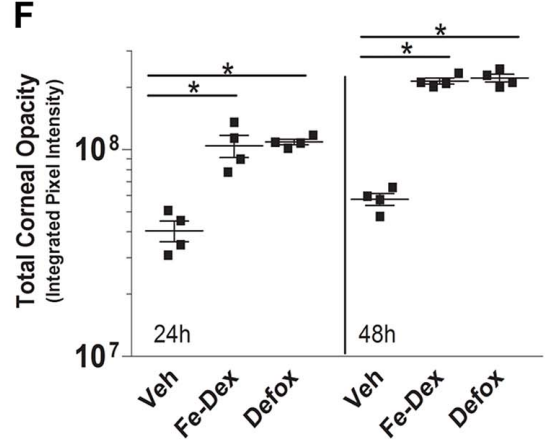

$\mathrm{H}$

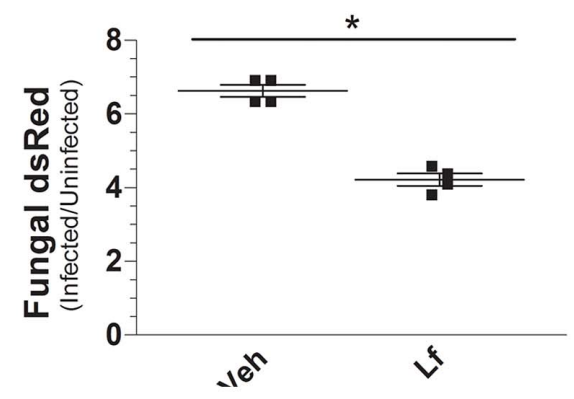

I.

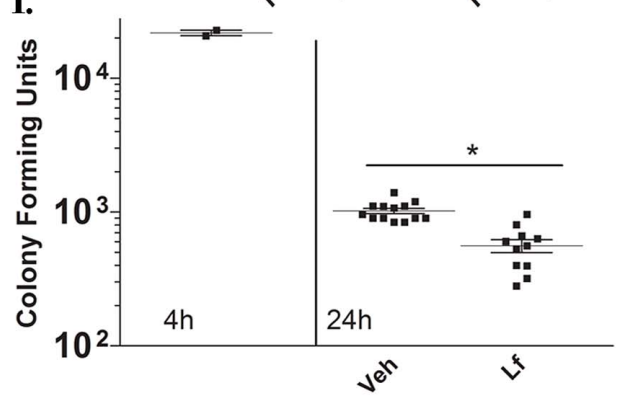

Figure 2. The effect of iron dextran, deferroxamine and lactoferrin on $\boldsymbol{A}$. fumigatus corneal infection. A. Serum iron levels $24 \mathrm{~h}$ after corneal infection. C57BL/6 mice were pre-treated at day -2, and day-1 with I.P. injections of iron-dextran (Fe-Dextran) or deferroxamine (Defox), and serum iron levels were measured by spectrophotometry (data are mean $+/-$ SD of 5 mice per group). B. Fungal growth (dsRed A. fumigatus) and corneal opacity in mice given Fe-Dextran or Defox. C. Metamorph image analyses of dsRed fluorescence. D. Colony forming units (CFU) per eye at $4 \mathrm{~h}$ and $48 \mathrm{~h}$ post-infection E,F. Metamorph image analyses of percent $(\mathbf{E})$ and total $(\mathbf{F})$ corneal opacification. G-I: Effect of lactoferrin on fungal growth. C57BL/6 mice were infected with $A$. fumigatus dsRed conidia, and given topical lactoferrin $(10.4 \mu \mathrm{g})$ at 0 and $6 \mathrm{~h}$ post-infection. Corneas were examined after 24 h. G: representative images; H. image analysis of dsRed expression, and I. CFU per eye. Panels B and G show representative images, and data points in panels $\mathrm{C}-\mathrm{F}, \mathrm{H}, \mathrm{I}$ represent individual corneas. All panels show representative data from one experiment except for panels $\mathrm{D}$ and I which show pooled data from repeat experiments. These experiments were repeated three times with similar results. doi:10.1371/journal.ppat.1003436.g002

with $\Delta$ sidD mutants had significantly lower CFU compared with WT $A$. fumigatus, whereas $\Delta$ sid $G$ were not significantly different. Corneal opacification scores reflected the CFU data, with $\Delta$ sidD but not $\Delta s i d G$ mutants having significantly less opacification than WT A. fumigatus (Figure 4C-E). These findings indicate that sidD-mediated synthesis of FusC rather than sidG-mediated synthesis of TAFG is necessary and sufficient to support fungal growth in vivo. Figure $\mathbf{S 1}$ shows that corneas infected with the complemented strains $\operatorname{sidF}^{\mathbf{R}}$ and $\operatorname{sidD}^{\mathbf{R}}$ show no phenotypic differences from the WT parental strain. Figure $\mathbf{S} 2$ shows that both intracellular and extracellular siderophore mutants of Alternaria brassicicola also have impaired growth during infection. Together, these data clearly demonstrate that extracellular siderophores are essential for both Aspergillus and Alternaria growth during tissue infection, and that even though TAFC is reportedly more stable [13], FusC production is sufficient to maintain fungal growth in vivo.

Mevalonate incorporation into extracellular siderophores is required for fungal infection

Fungal extracellular siderophore biosynthesis requires HMG$\mathrm{CoA}$ reductase-dependent synthesis of the precursor mevalonate [14]. The Aspergillus genes sidI and sidH encode a CoA-ligase and an enoyl-CoA-hydratase, respectively, which convert mevalonic acid to anhydromevalonyl CoA and incorporate this precursor through the sidF-D-G pathway into the structure of fusarinine $\mathrm{C}$ and TAFG (Figure 5A) [13,14]. 
A

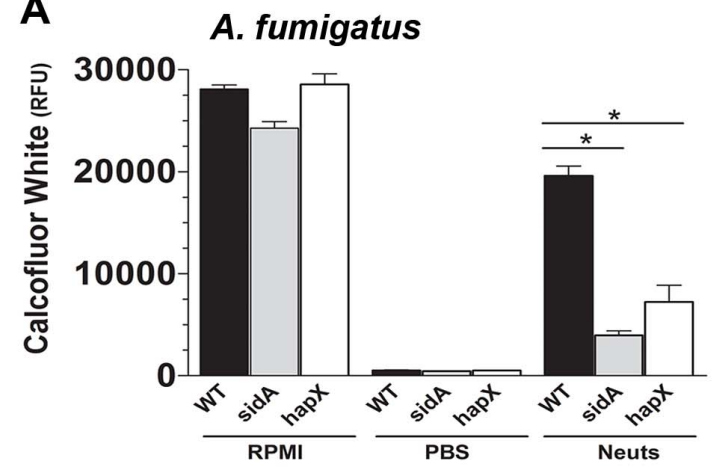

B

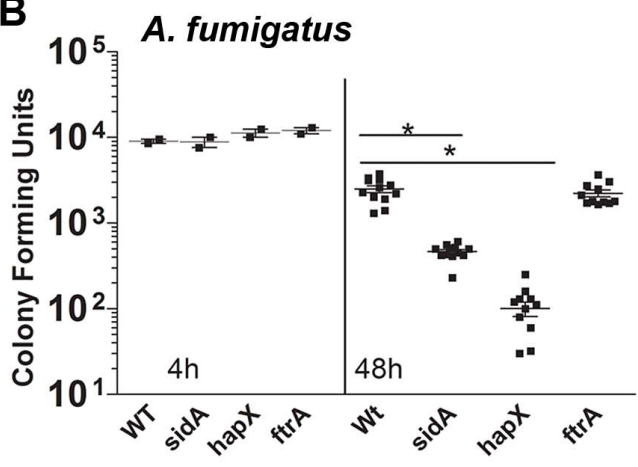

C A. fumigatus

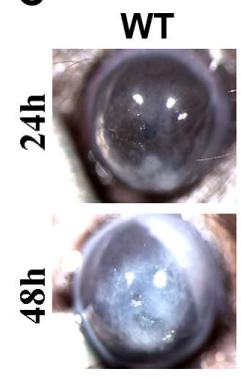
AsidA
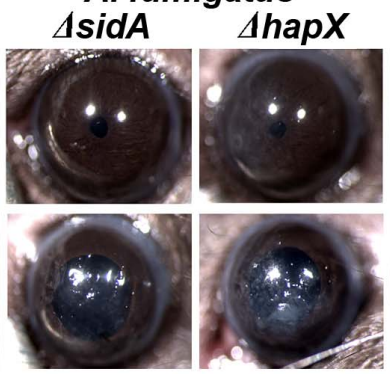

$\Delta$ ftrA

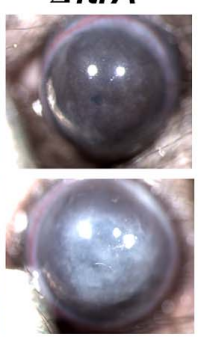

$\mathbf{F}$

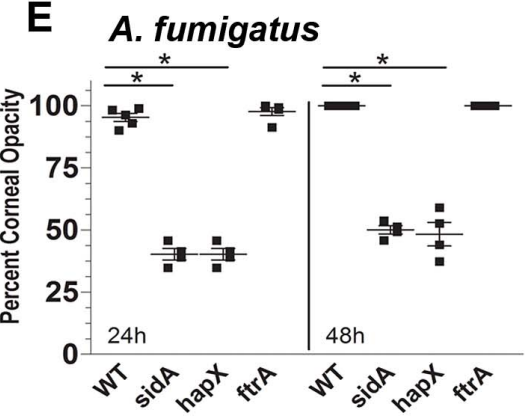

D

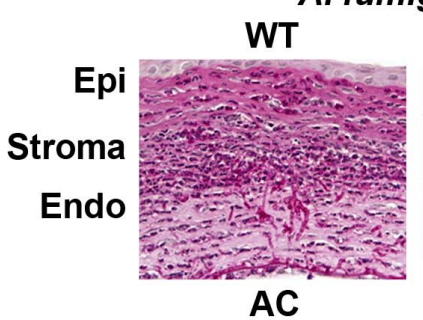

A. fumigatus
H

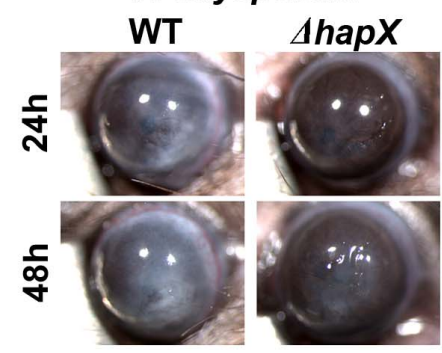

F A. fumigatus

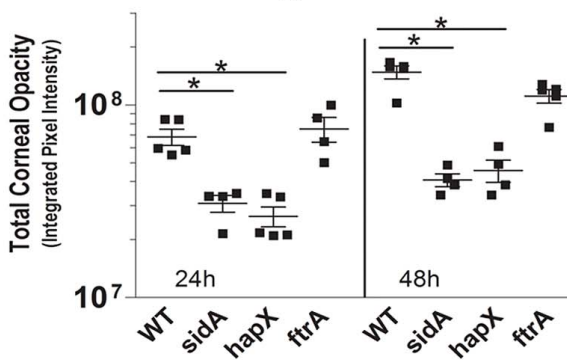

I

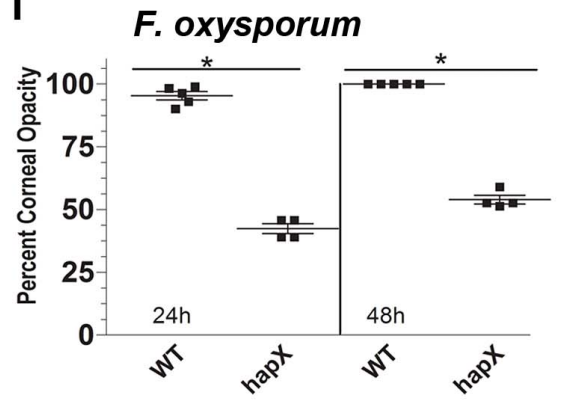

AC
G

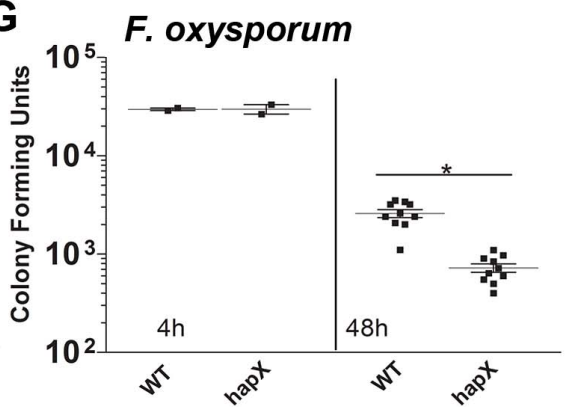

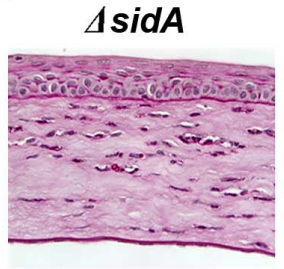
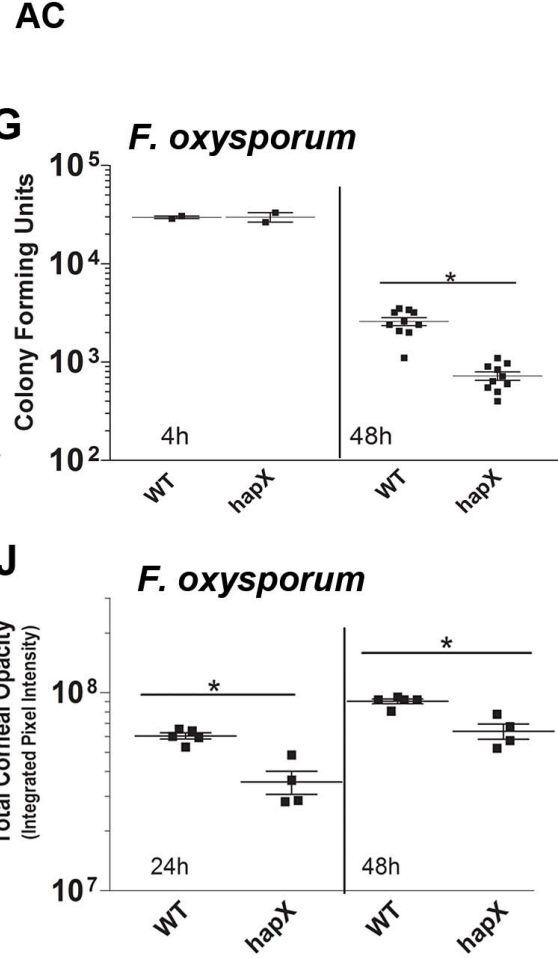

Figure 3. Susceptibility of $A$. fumigatus siderophore and iron acquisition mutants in fungal keratitis. A. Growth $A$. fumigatus $\Delta$ sidA and $\Delta$ hapX mutants after incubation with human neutrophils; fungal mass was quantified using calcofluor white and fluorometry. (Mean $+/-$ SD of 5 replicate wells.) B-F: A. fumigatus corneal infection; G-J F. oxysporum corneal infection. B: CFU per eye of C57BL/6 mice $48 \mathrm{~h}$ after intrastromal infection with A. fumigatus $\Delta$ sidA, $\Delta$ hapX, or $\Delta f t r A$ mutants. C. Representative eyes showing corneal opacification at $24 \mathrm{~h}$ and $48 \mathrm{~h}$. D. Representative corneal sections stained with periodic acid-schiff and hematoxylin (PASH). E. Quantification of percent corneal opacity and F. Total cornea opacity. G-J. C57BL/6 corneas were infected with F. oxysporum $\Delta$ hapX and the parent strain. G. CFU per eye at $4 \mathrm{~h}$ and $48 \mathrm{~h}$ post-infection H. Representative eyes showing corneal opacification. I. Quantification of percent corneal opacity J. Total cornea opacity. Data points represent individual corneas. All panels show representative data from one experiment except for panels B and $G$ which show pooled data from repeat experiments. Similar results were found in three repeat experiments. Abbreviations: Neuts- neutrophils, epi-epithelium, endo-endothelium, AC-anterior chamber. doi:10.1371/journal.ppat.1003436.g003

To determine if this pathway is essential for fungal growth during tissue infection, C57BL/6 mice were infected with $A$. fumigatus mutant strains $\Delta$ sidH and $\Delta$ sidI and examined as before. Figure 5B shows that mice infected with $\Delta$ sidH or $\Delta s i d I$ exhibit significantly less CFU than mice infected with the WT strain, indicating that mevalonate incorporation into extracellular siderophores is essential for fungal growth during tissue infection. Further, mice infected with either $\Delta$ sidH or $\Delta$ sidI exhibit 
A

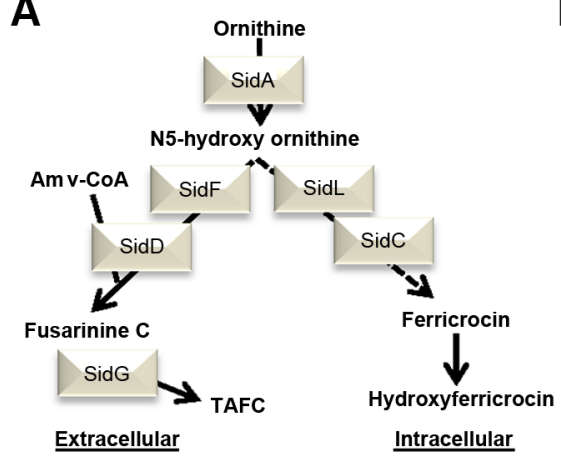

B

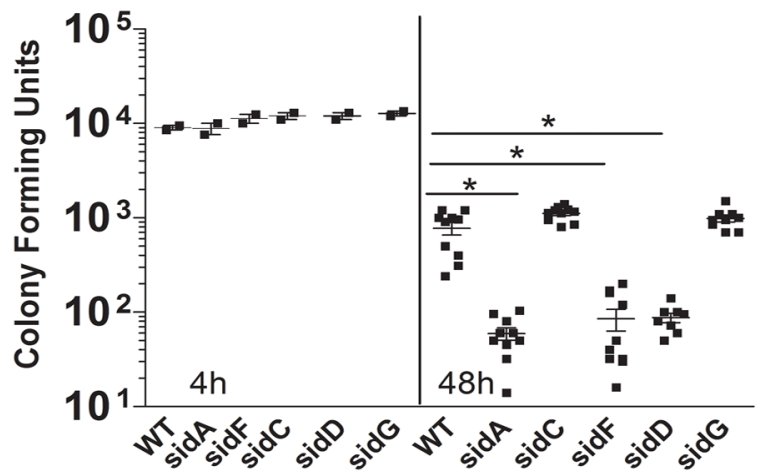

C

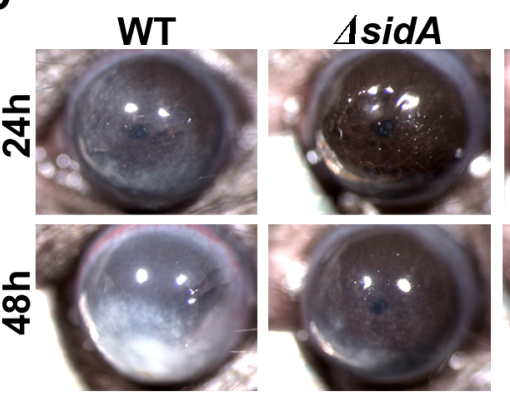

AsidF

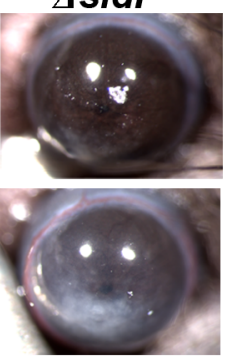

AsidC

D

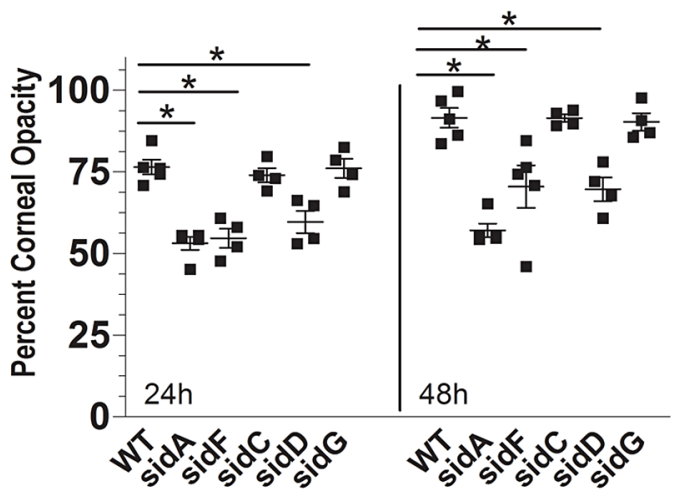

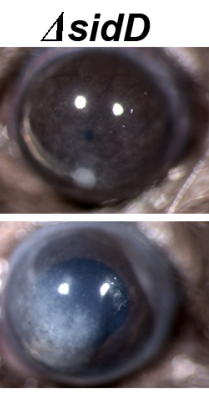
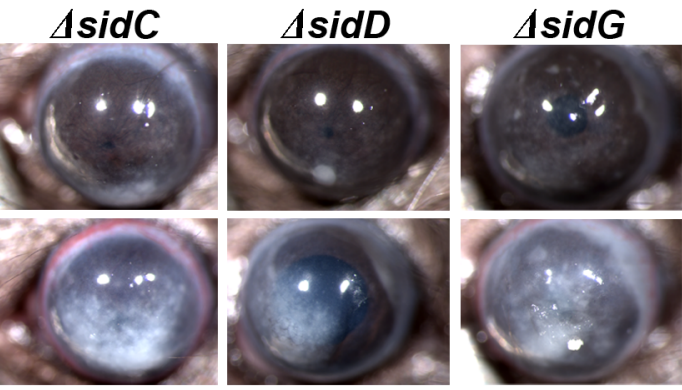

$E$

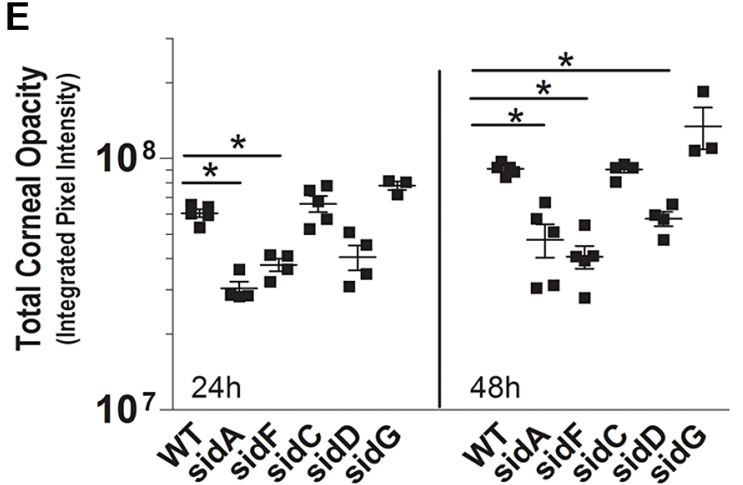

Figure 4. The role of intracellular and extracellular siderophores in $A$. fumigatus corneal infection. A. Biosynthesis of $A$. fumigatus siderophores. B-E. C57BL/6 mice infected intrastromally with $A$. fumigatus mutant strains $\Delta$ sidA, $\Delta s i d C, \Delta s i d F, \Delta s i d D$, or $\Delta s i d G$. B. CFU per eye at $4 \mathrm{~h}$ and $48 \mathrm{~h}$ post-infection. C. Representative eyes showing corneal opacity. D. Quantification of percent corneal opacity and E. total cornea opacity. B, D, E: Data points represent individual corneas. All panels show representative data from one experiment except for panel B which shows pooled data from repeat experiments. Similar results were found in 3 repeat experiments. Abbreviations: Amv- anhydromevalonyl; TAFC: tri-acetyl fusarinine C. doi:10.1371/journal.ppat.1003436.g004

significantly less cornea opacity at all time-points compared to mice infected with WT $A$. fumigatus (Figure 5C-E). Figure S1 shows that corneas infected with the complemented $\Delta$ sidH strain $\operatorname{sid}^{\mathbf{R}}$ or the complemented $\Delta s i d I$ strain $\operatorname{sidI}^{\mathbf{R}}$ show no significant difference in opacification or CFU as the WT parental strain."

Lipocalin-1 sequesters fungal siderophores and restricts fungal growth during infection

Humans produce two lipocalins with siderophore binding activity [26]. Lipocalin-1 (Lcn-1) binds to a wide range of bacterial and fungal hydroxamate-type siderophores [17], whereas Lcn-2 binds catechol-type bacterial siderophores but not fungal siderophores [31,32]. We therefore examined the role of Lcn-1 on $A$. fumigatus using the same assays as above. Figure 6B shows significantly less fungal growth incubated with $40 \mu \mathrm{g} / \mathrm{ml}$ or $4 \mu \mathrm{g} /$ $\mathrm{ml}$ Lcn-1 than in RPMI alone, and that growth of $A$. fumigatus in the presence of neutrophils and $4 \mu \mathrm{g} / \mathrm{ml} \mathrm{Lcn-1} \mathrm{was} \mathrm{significantly}$ less than with neutrophils alone or Lcn-1 alone. Figures 6C-E show significantly less fungal dsRed and CFU in mice given topical Lcn-1 $(16 \mu \mathrm{g} / 8 \mu \mathrm{l})$ at $0 \mathrm{~h}$ and $6 \mathrm{~h}$ after infection compared with infected mice not given Lcn-1. These findings indicate that topical Lcn-1 inhibits fungal growth in vivo, presumably by sequestering fungal siderophores.

\section{Topical simvastatin and deferiprone inhibit fungal} infection

As shown in Figure 5, Aspergillus SidI and SidH proteins incorporate mevalonate into the structure of extracellular siderophores and are essential for fungal growth in the cornea. Also, HMG-CoA reductase is required for mevalonate production and can be targeted by statins to inhibit siderophore biosynthesis $[14,33]$ (Figure 7A). To determine the effect of blocking this 
A

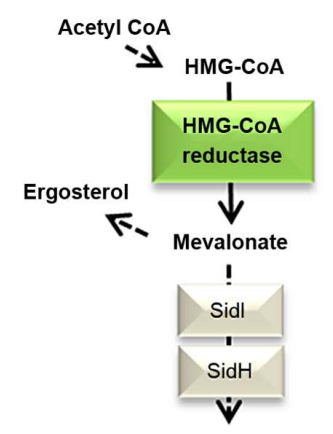

Anhydromevalonyl-CoA

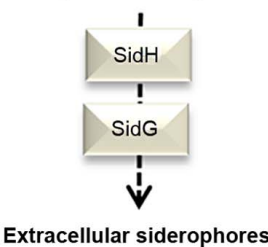

B

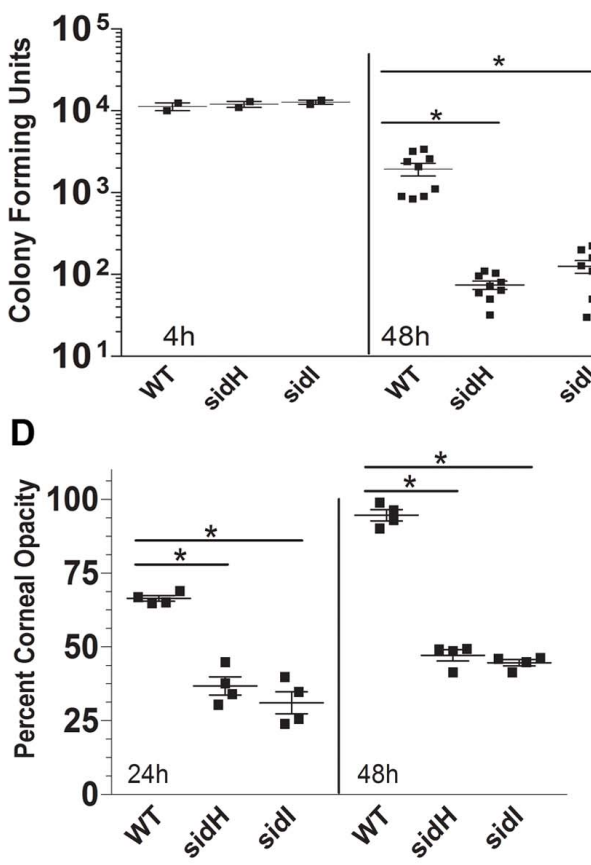

C

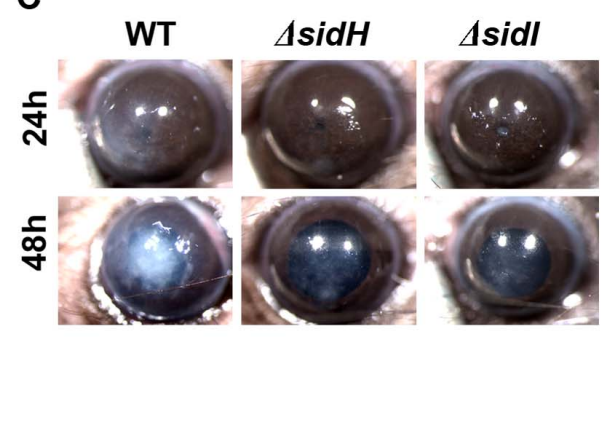

E

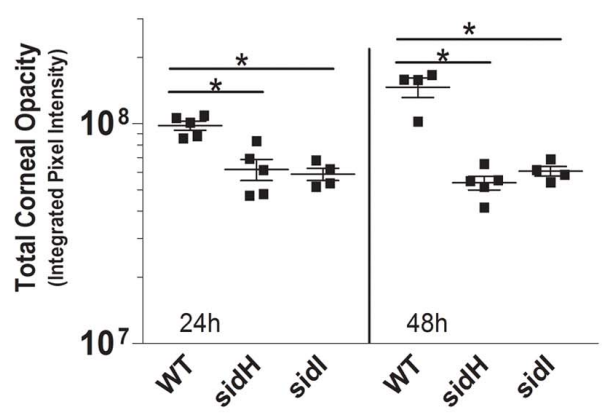

Figure 5. Role of mevalonate pathway for extracellular siderophores in $A$. fumigatus corneal infection. A. Mevalonate extracellular siderophore biosynthesis pathway. B-E. C57BL/6 mice infected intrastromally with $A$. fumigatus $\Delta$ sidH and $\Delta$ sidl mutant strains. B. CFU per eye at $4 \mathrm{~h}$ and $48 \mathrm{~h}$ post-infection. C. Representative eyes showing corneal opacity D. Quantification of percent corneal opacity and E. total corneal opacity. B, $D, E$ : Data points represent individual corneas. All panels show representative data from one experiment except for panel B which shows pooled data from repeat experiments. Similar results were found in 3 repeat experiments. Abbreviations: HMG-CoA: 3-hydroxy-3-methyl-glutaryl-CoA. doi:10.1371/journal.ppat.1003436.g005

pathway on fungal growth, A. fumigatus and $F$. oxysporum were incubated with the HMG-CoA reductase inhibitors simvastatin and lovastatin, or with the iron chelators deferiprone and deferroxamine.

As shown in Figure 7B, there was significantly less growth of $A$. fumigatus following $16 \mathrm{~h}$ incubation in SDB media with simvastatin, lovastatin and deferiprone, but not deferroxamine, thereby demonstrating a direct effect of statins and deferiprone on fungal growth. Similar results were obtained with Fusarium oxysporum (Figure 7G). Interestingly, a statin dependent dose curve was observed with $A$. fumigatus when exposed to simvastatin or lovastatin (Figure 7B), however, a dose curve was only observed when Fusarium was treated with simvastatin not lovastatin (Figure 7G). This observation likely reflects differences in the pharmacokinetics of different statins and their ability to penetrate into the fungal cytoplasm and inhibit HMG-CoA reductase of multiple fungal genera and species [33].

To ascertain if these agents enhance fungal sensitivity to killing by neutrophils, $A$. fumigatus conidia were cultured for $4-6 \mathrm{~h}$ in SDB media, washed, and incubated a further $16 \mathrm{~h}$ with human neutrophils in RPMI media containing simvastatin, lovastatin or deferiprone. As shown in Figure 7D, fungal growth was significantly less when incubated with neutrophils and simvastatin, lovastatin, and deferiprone compared with neutrophils alone, whereas there was no significant difference in the presence of deferroxamine. The inhibitory effect of $1 \mu \mathrm{M}$ statins on fungal growth shown in panel B was not observed in this assay, most likely due to siderophore production during the $4-6 \mathrm{~h}$ growth in the absence of statins.

To ascertain if statins can restrict fungal growth during infection, mice were infected intrastromally with A. fumigatus, and given topical simvastatin, deferiprone, or deferroxamine at the time of infection and after $6 \mathrm{~h}$. At $24 \mathrm{~h}$ post-infection, mice eyes were imaged for corneal opacity and fungal dsRed, and processed for fungal CFU. Importantly, unlike prior experiments, which examined CFU at the $48 \mathrm{~h}$ time-point, at $24 \mathrm{~h} \mathrm{CFU}$ in infected eyes does not decrease unless treated with anti-microbial agents. Therefore, in this $24 \mathrm{~h}$ assay and unlike prior experiments, vehicle-treated CFU do not decrease but instead represent the maximum fungal CFU value per assay. As shown in Figure 7E and $\mathbf{F}$, mice treated with simvastatin or deferiprone exhibited significantly less fungal mass compared to vehicle-treated mice, which was further decreased when given both compounds. Conversely, mice given simvastatin together with deferroxamine had significantly higher fungal mass than mice given simvastatin alone, indicating that exogenous deferroxamine counters the inhibitory activity of statins. Figure 7G shows similar responses when CFU were measured $24 \mathrm{~h}$ post infection, with significantly less CFU in mice given simvastatin, deferiprone or both, and partial reversal of the inhibitory effect of simvastatin when mice also received topical deferroxamine, indicating that simvastatin is targeting siderophore biosynthesis in addition to ergosterol synthesis in vivo. Mice treated with deferroxamine alone showed elevated fungal mass (Figure 7E) compared with vehicle-treated mice, but at this time point, CFU values were not significantly different from vehicle-treated mice (Figure 7F). Taken together, these findings clearly demonstrate that topical statins and iron chelation can block fungal growth during infection.

\section{Discussion}

Previous work in our laboratory demonstrated that fungal antioxidative responses are essential for survival during tissue infection, and that fungal growth can be inhibited in vivo by 
A

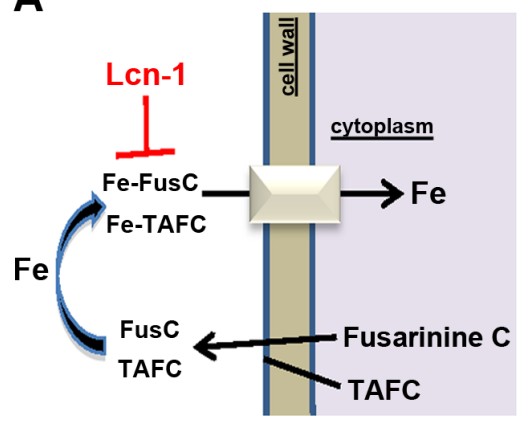

B

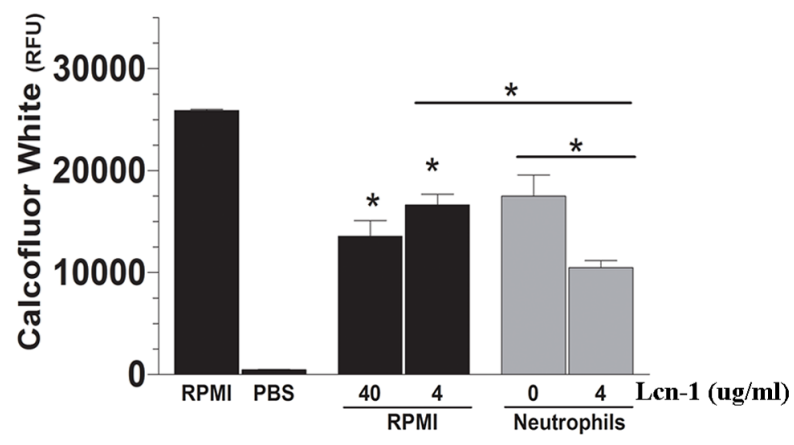

C

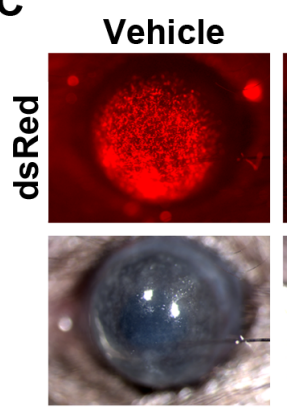

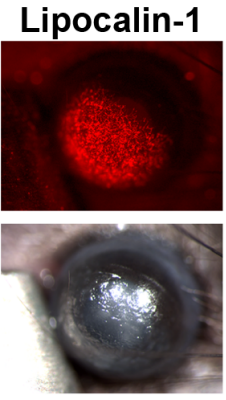

D

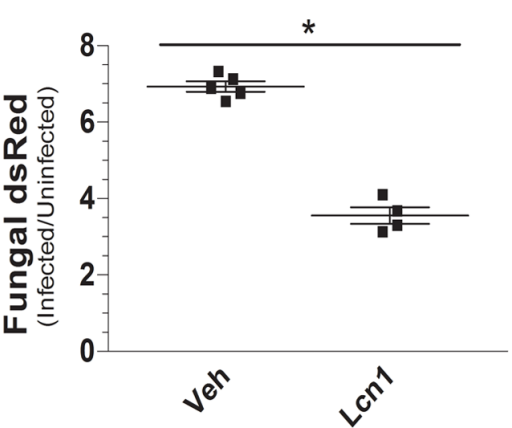

E

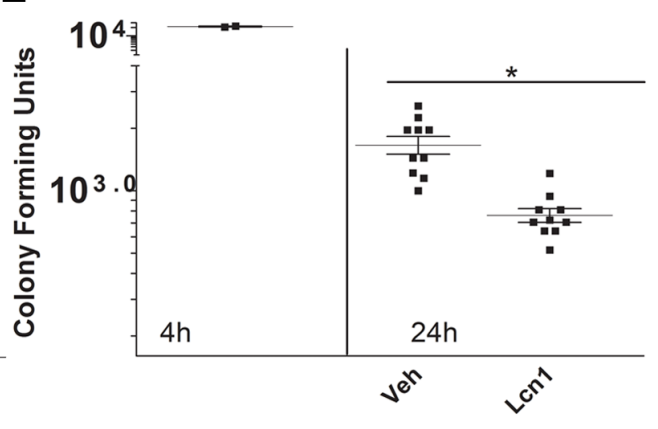

Figure 6. Effect of exogenous lipocalin-1 in A. fumigatus corneal infection. A. Pathway showing Lcn-1 sequestration of fungal siderophores. B. Growth of $A$. fumigatus incubated with recombinant human Lcn-1 in the absence (black bars) or presence (gray bars) of human neutrophils determined by calcofluor white binding and quantification using fluorometry (data are mean +/-SD of five replicate wells). C-E: C57BL/6 mice were given topical Lcn-1 $(16 \mu \mathrm{g})$ at 0 and $6 \mathrm{~h}$ post-infection with $A$. fumigatus dsRed. C. Representative corneas; D. Metamorph image analysis showing fungal dsRed expression and E. CFU per eye. Data points represent individual corneas. All panels show representative data from one experiment except for panel $\mathrm{E}$ which shows pooled data from repeat experiments. Similar results were found in two repeat experiments. Abbreviations: FusCfusarinine C, TAFC- tri-acetyl fusarinine C, Lcn-lipocalin.

doi:10.1371/journal.ppat.1003436.g006

targeting fungal thioredoxin [8]. As thioredoxin-regulated peroxiredoxases, catalases, and other antioxidants require iron to quench reactive oxidants [9,34], the current study examined the role of host iron sequestration and fungal iron acquisition during infection. In examining endogenous iron levels, we found a twofold reduction in serum iron levels following fungal infection which correlated with elevated expression of iron-chelating proteins, heme-binding and siderophore-sequestering proteins in infected corneas, and systemic induction of hepatic hepcidin. Expression of these iron-sequestering proteins was dependent on Dectin-1, which we and others showed is important in recognizing $\beta$-glucan on germinating conidia $[23,35,36]$. Expression of these proteins was also dependent on IL-6, which has been shown to induce liver hepcidin and reduce systemic iron levels [15].

In this iron-restricted environment, we show that fungal siderophores are essential for microbial growth and survival. This notion is supported by our findings that siderophore mutants are unable to grow in the cornea, and that exogenous iron chelators or inhibitors of fungal siderophore biosynthesis impair fungal growth both in vitro when incubated with human neutrophils, and in vivo in a murine model of fungal infection. Given that the siderophore biosynthesis pathway is highly conserved in filamentous fungi [12], our findings are very likely relevant to fungal infections of other tissues in addition to other fungal pathogens. Figure 8 illustrates the fungal siderophore biosynthesis pathway and highlights the key findings in this study.

Firstly, we identified that both Aspergillus and Fusarium require the transcription factor HapX to sense and respond transcription- ally to low iron levels and survive during infection. Further, we show that $A$. fumigatus strains $\Delta s i d F, \Delta s i d D, \Delta s i d H$, or $\Delta s i d I$, that do not express the extracellular siderophores are attenuated during infection of the cornea, whereas based on the absence of a phenotype with $\Delta$ sidC mutants, there is no apparent role for intracellular siderophores. We show that production of extracellular siderophores is also required for infection with Alternaria. These findings are consistent with an essential role for HapX and extracellular siderophores during experimental A. fumigatus lung infection $[11,14,30]$, the requirement of HapX for Fusarium infection of tomato plants and immunosuppressed mice [37], and the requirement of siderophores for Alternaria infection in maize [38]. Interestingly, iron starvation also activates the transcription factor AcuM, which increases HapX expression and down-regulates the iron-repressing transcription factor SreA, resulting in fungal iron acquisition [39]. The role of AcuM and SreA during corneal infection has yet to be determined.

In the present study, we also examined the potential for statins to inhibit fungal HMG-CoA reductase, which is required for mevalonic acid production and extracellular siderophore biosynthesis [14]. Consistent with a report on pulmonary aspergillosis [14], we used mutants to demonstrate that SidI and SidHdependent mevalonic acid incorporation into extracellular siderophores is essential for infection of the cornea. Further, we show that simvastatin and lovastatin inhibit fungal growth in vitro, and also enhance growth inhibition by human neutrophils. Consistent with the difference in statin activity in treating hypercholesterolemia [40], simvastatin exhibited 10-fold greater inhibitory activity 
A

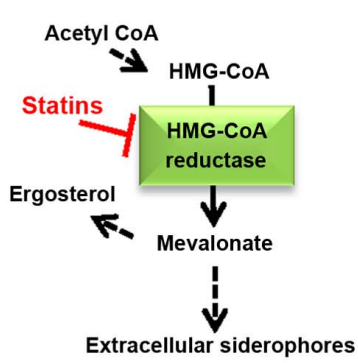

B

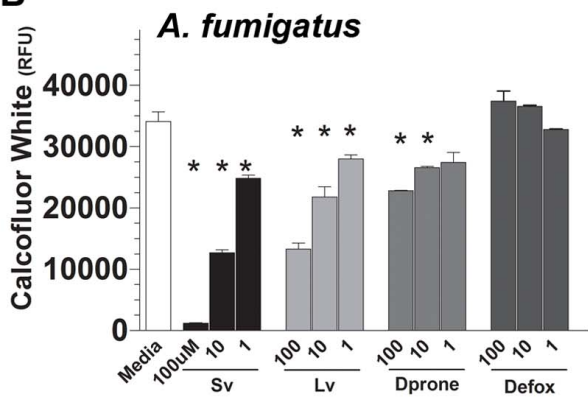

C

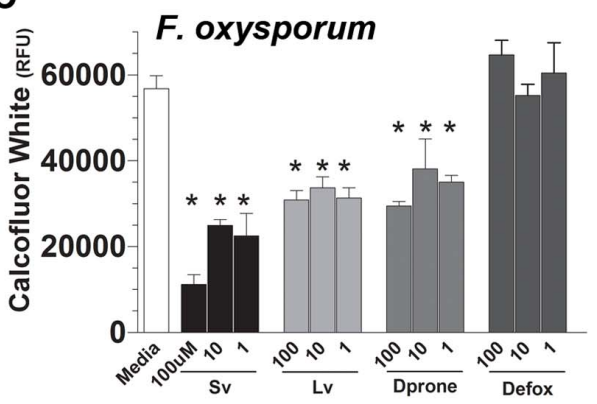

D

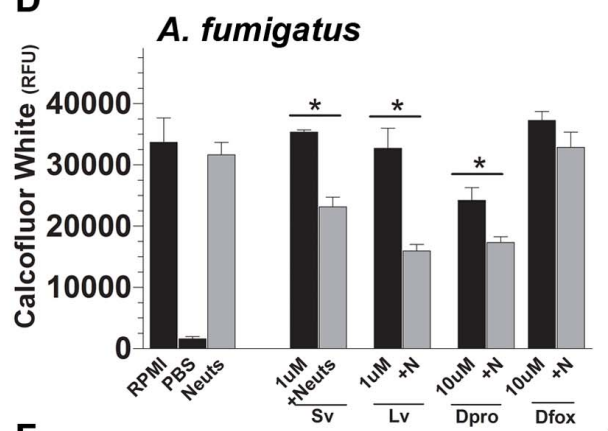

$\mathbf{F}$

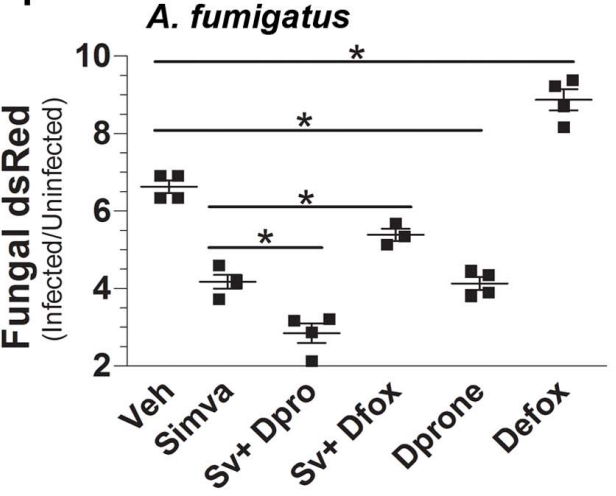

E

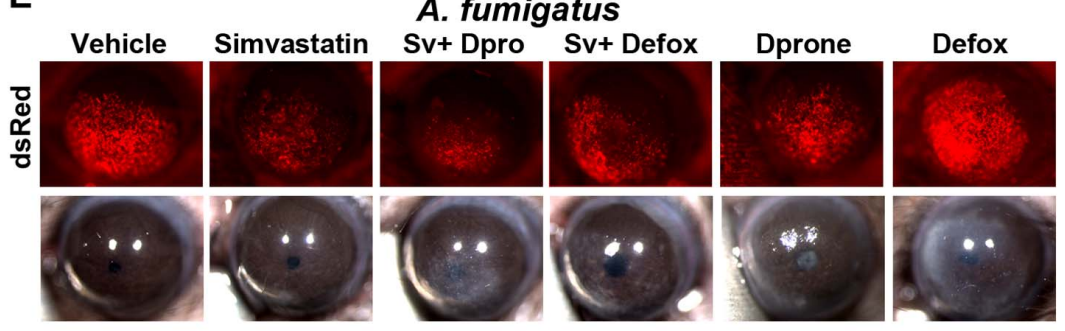

G

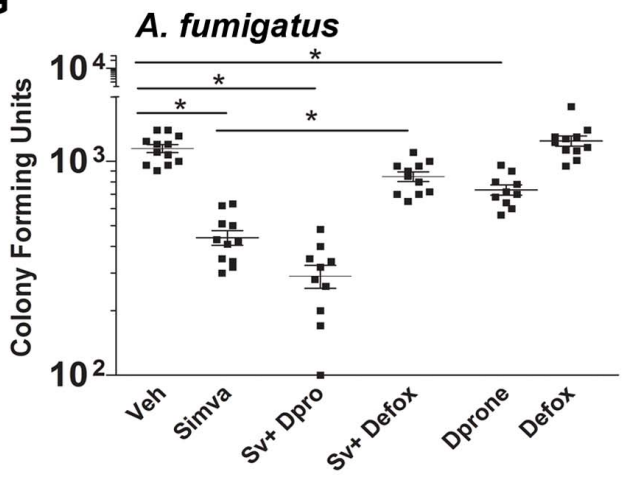

Figure 7. Effect of topical simvastatin and deferiprone on fungal infection. A. Statin targeting of fungal HMG-CoA reductase in siderophore biosynthesis. B, C. Effect of statins and iron chelators on growth of $A$. fumigatus and $F$. oxysporum in vitro. B. Simvastatin, lovastatin, deferiprone, or deferroxamine were added to growing cultures of $A$. fumigatus or C. F. oxysporum for 16 h, and hyphal growth was quantified by calcofluor white. D. Growth of $A$. fumigatus incubated with these compounds in the absence (black bars) or presence (gray bars) of human neutrophils by calcofluor white quantification (data are mean $+/-$ SD of five replicate wells) E. C57BL/ 6 mice were infected with A. fumigatus and at 0 and $6 \mathrm{~h}$ post-infection $13.4 \mu \mathrm{g}$ of simvastatin (Sv), deferiprone $(11.1 \mu \mathrm{g})$, deferroxamine $(52.5 \mu \mathrm{g})$, Sv+ deferiprone, or Sv+ deferroxamine was applied topically to infected corneas and eyes were imaged at $24 \mathrm{~h}$ post-infection. F. Metamorph image analysis was used to quantify fungal dsRed expression and G. eyes were homogenized for CFU analysis. B-D: data are mean +/-SD of five replicate wells; F,G: data points represent individual corneas. All panels show representative data from one experiment except for panel $G$ which shows pooled data from repeat experiments. Similar results were found in three repeat experiments. Abbreviations: HMG- 3-hydroxy-3-methyl-glutaryl-CoA, Sv-simvastatin, Lv-lovastatin, Dprone- deferiprone, Defoxdeferroxamine.

doi:10.1371/journal.ppat.1003436.g007

than lovastatin in restricting fungal growth in vitro. During infection, topical application of simvastatin inhibited fungal growth that was only partially reversed by exogenous siderophores, indicating that statins function in vivo by inhibiting both fungal siderophore and ergosterol biosynthesis. The fungicidal activity of statins has been reported for Aspergillus, Fusarium, Mucorales, and Candida [41-43]. However, to our knowledge, this is the first study to clearly demonstrate a therapeutic effect of statins in an experimental fungal infection. In contrast to systemic statin treatment, topical application is likely to have minimal risk of side effects [33]. Future studies will examine if statins can restrict fungal growth at later stages of infection.
In addition to showing inhibition of siderophore biosynthesis, the current study demonstrates that exogenous lipocalin-1 impairs fungal growth in the presence of human neutrophils and during infection. This finding is consistent with the reported role for Lcn1 in sequestering fungal siderophores, including TAFC [17]. The affinity of Lcn-1 for fungal siderophores is similar to that of fungal siderophore receptors [17], and therefore at high concentrations Lcn-1 could sequester siderophores from fungal receptors. Further, it is likely that Lcn-1-siderophore complexes are internalized through the lipocalin-interacting membrane receptor (LIMR) resulting in siderophore degradation $[44,45]$. Endogenous Lcn-1 is abundant in human tears $(3 \mathrm{mg} / \mathrm{ml})$, nasal mucosa, and tracheal 


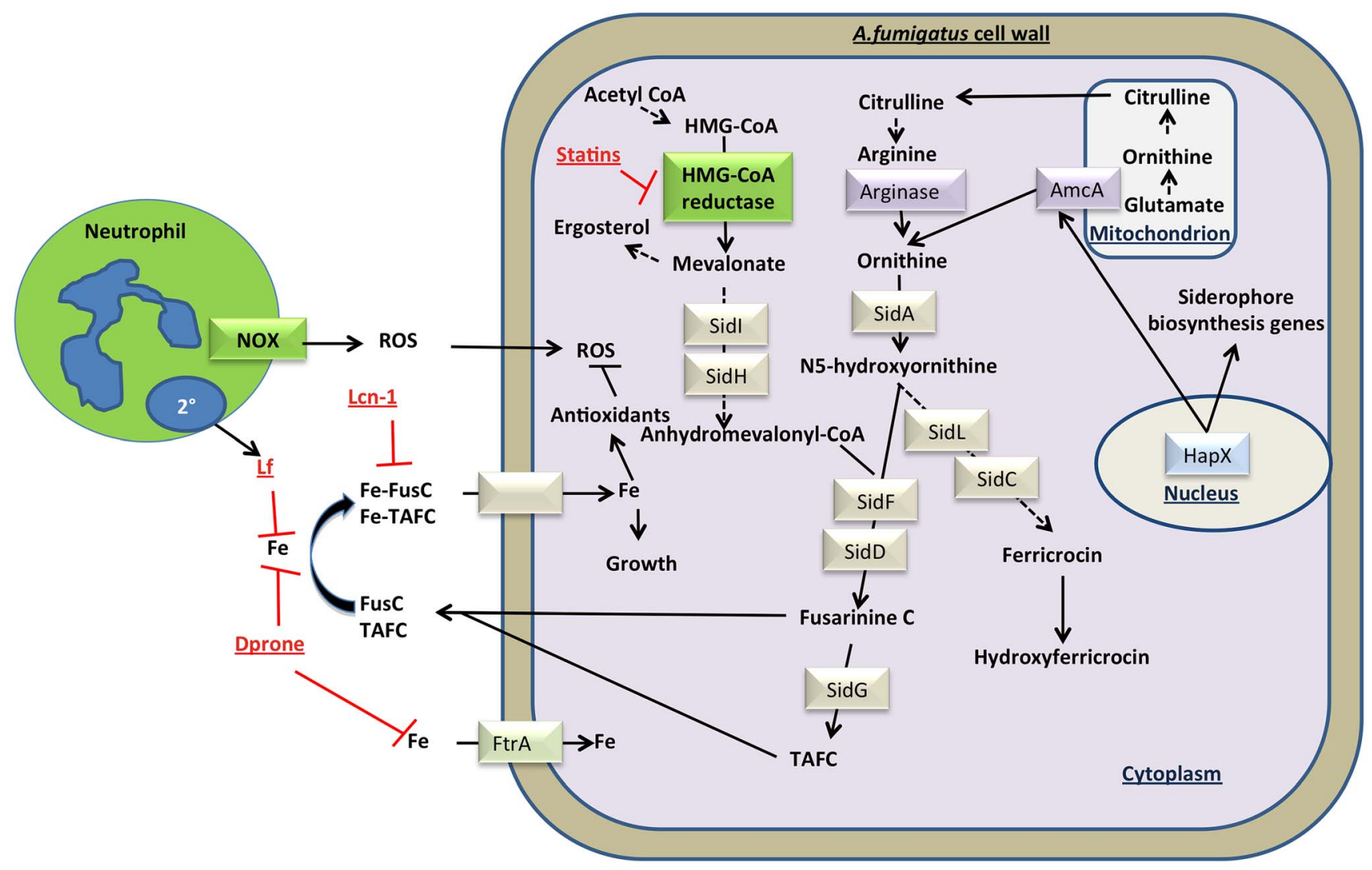

Figure 8. The battle for iron between the mammalian host and fungi. Defox- deferroxamine, Dprone- deferiprone, Fe-iron, Fus Cfusarinine C, HMG-CoA- 3-hydroxy-3methylglutaryl-coenzyme, Lcn-1- lipocalin 1, Lf- lactoferrin, NOX- nicotinamide adenine dinucleotide phosphate oxidase, ROS-reactive oxygen species, TAFC- triacetyl fusarinine C. doi:10.1371/journal.ppat.1003436.g008

secretions where it can function prophylactically to prevent mucosal fungal infections $[17,46]$. However, as we now show that human neutrophils express Lcn-1, it is possible that Lcn-1 also has a protective role during active infection.

In addition to targeting siderophores, we showed that reducing local tissue iron concentrations by topical application of the iron chelating protein lactoferrin restricts fungal growth in vivo. This finding is consistent with the reported role for lactoferrin in blocking conidia germination in vitro by human neutrophils [47], and suggests that in vivo, neutrophil-derived lactoferrin restricts the growth of conidia and hyphae by binding free iron. Given that siderophores exhibit a higher affinity for iron than lactoferrin [12], it is likely that fungal siderophores can acquire iron from lactoferrin during infection; however, the rate of siderophore iron acquisition in a lactoferrin-rich environment is likely slower than in the absence of lactoferrin given the scarcity of free iron or ironbound to lower affinity biomolecules.

Similarly, we showed that the iron chelator deferiprone sensitizes fungi to human neutrophils and blocks fungal infection. However, deferiprone is also a very small molecule (MW=140 g/mole), approximately 600-fold smaller than lactoferrin, and is therefore released from tissues more readily than lactoferrin, resulting in both iron sequestration and depletion from infected tissues [48]. Deferiprone has been used effectively and safely to lower iron levels in patients with hemochromatosis [48], and although widely utilized in Europe, it is not currently licensed in the USA. Importantly, deferiprone, unlike deferroxamine, is not a xenosiderophore [28] and is not associated with an increased risk of fungal and bacterial infections [49-57].
Iron chelators have been used to treat Aspergillus and Rhizopus infections in mice [57-60], and a clinical trial examined the potential of the iron chelator deferasirox to enhance the efficacy of liposomal amphotericin B to treat mucormycosis (the DEFEAT Mucor study). However, the trial was unsuccessful due to an unexpected increased risk of death in patients receiving deferasirox adjunct therapy [61]. The DEFEAT Mucor study exhibited a limited sample size and imbalanced stratification of the sickest patients into the deferasirox treatment group and may not accurately reflect the potential of deferasirox to treat mucormycosis. However, systemic deferasirox treatment does cause side effects that include agranulocytosis and nephrotoxicity [62]. In the current study, we demonstrated that local (topical) application of iron chelators is both effective in inhibiting fungal growth and preventing corneal disease. As local administration is highly unlikely to cause systemic side effects, clinical studies using topical iron chelators and fungal iron acquisition inhibitors are unlikely to cause adverse reactions, especially if combination therapies targeting iron acquisition can use low drug concentrations. This approach could be used to treat fungal infections not only in the cornea, but also in other tissues that could be treated topically such as the tongue, skin, and nails.

In conclusion, we have identified host iron sequestration and fungal siderophore biosynthesis as essential mediators of fungal growth during infection. One approach to exploiting these findings is to chelate local iron at the infectious site utilizing deferiprone or lactoferrin. A second approach is to inhibit the ability of fungi to acquire iron utilizing the siderophore-binding protein Lcn-1 or the 
siderophore biosynthesis inhibitor simvastatin. However, the most efficacious strategy would likely involve the combination of iron chelation and inhibition of siderophore biosynthesis. In this study, we provide proof-of-concept that dual treatment with deferiprone and simvastatin further restricts fungal growth during infection. As both deferiprone and simvastatin, have a long history of safe use in patients, it is possible that these agents can be successfully utilized to treat a broad range of fungal infections.

\section{Materials and Methods}

\section{Use and source of animals}

All animals were treated in accordance with the guidelines provided in the Association for Research in Vision and Ophthalmology ARVO statement for the Use of Animals in Ophthalmic and Vision Research, and were approved by Case Western Reserve University IACUC. C57BL/ 6 mice (6-12 wk old) and IL$6^{-1-}$ mice on a C57BL/6 background were from the Jackson Laboratory (Bar Harbor, ME), Dectin-1 ${ }^{-1-}$ mice were kindly provided by Dr. Yoichiro Iwakura (University of Tokyo; Tokyo, Japan).

\section{Fungal strains and growth conditions}

Table 1 lists the genotype and phenotype of all strains utilized in this study. Aspergillus fumigatus was cultured on Vogel's minimal media (VMM) $+2 \%$ agar and Fusarium oxysporum lycopersici was cultured on potato dextrose agar (PDA). Alternaria brassicicola was cultured in complete media as described previously [38]. All solid media used in this study were supplemented with $10 \mathrm{mM} \mathrm{FeSO}$ to enhance conidia production by siderophore mutants. For neutrophil-fungus incubation assays, all fungi were grown in RPMI media w/o $\mathrm{FeSO}_{4}$ supplementation. The Alternaria brassicicola $\Delta n p s 2, \Delta n p s 6$, and $\Delta n p s 2 / 6$ strains were kindly provided by Dr. B. Gillian Turgeon (Cornell University, Ithaca, NY).

\section{Mouse model of Aspergillus and Fusarium keratitis}

Aspergillus and Fusarium strains were cultured as described above for 2-3 days, and fresh conidia were disrupted with a bacterial Lloop, harvested in $5 \mathrm{ml} \mathrm{PBS}$, and filtered through sterile PBSsoaked cotton gauze in a $10 \mathrm{ml}$ syringe to obtain pure conidial suspensions. Conidia were quantified using a hemocytometer and adjusted in PBS to a final stock solution of 15-20,000 conidia/ $\mu$ l. Mice were anaesthetized with $1.25 \% 2,2$, 2-tri-bromoethanol in PBS. The corneal epithelium was abraded using a 30-gauge needle, through which a $2 \mu \mathrm{l}$ injection containing conidia was released into the corneal stroma using a 33-gauge Hamilton syringe. Mice were examined daily under a stereomicroscope for corneal opacification, and quantified by image analysis using Metamorph software as described [8,23]. At each time point, animals were euthanized by $\mathrm{CO}_{2}$ asphyxiation, and eyes were either placed in $10 \%$ formalin and embedded in paraffin, sectioned at $5 \mu \mathrm{m}$ intervals and stained with periodic acid Schiff and Hematoxylin (PASH), or were placed in $1 \mathrm{ml}$ of sterile PBS, homogenized and colony forming units (CFU) were quantified by manual count.

\section{Topical and systemic drug delivery}

Compounds were suspended in a commercial eye drop formulation (Alcon laboratories) or in PBS, and $8 \mu \mathrm{l}$ was applied topically at $0 \mathrm{~h}$ and $6 \mathrm{~h}$ post-infection. Lactoferrin $(1.25 \mathrm{mg} / \mathrm{ml})$, deferiprone $(10 \mathrm{mM})$, deferroxamine $(10 \mathrm{mM})$, and simvastatin $(4 \mathrm{mM})$ were purchased from Sigma Aldrich (St.Louis, MO). E.coli-expressed recombinant human lipocalin-1 was purified as described previously [17] and applied topically at $2 \mathrm{mg} / \mathrm{ml}$. Iron- dextran and deferroxamine were purchased from Sigma and administered systemically to mice by daily intraperitoneal injections of $5 \mathrm{mg}$ starting at day -2 until mice were euthanized. All animals were bred under specific pathogen-free conditions and maintained according to institutional guidelines.

\section{Quantification of Aspergillus fungal mass and colony forming units (CFUs)}

Growth of the RFP expressing A. fumigatus strain in the cornea was detected by fluorescent microscopy and quantified by Metamorph image analysis [8,23]. For assessment of fungal viability, whole eyes were homogenized under sterile conditions in $1 \mathrm{ml}$ PBS, using the Mixer Mill MM300 (Retsch) at $33 \mathrm{~Hz}$ for $4 \mathrm{~min}$. Subsequently, $100 \mu \mathrm{l}$ aliquots were plated onto bacteriologic-grade Sabouraud dextrose agar plates, incubated for $24 \mathrm{~h}$ at $37^{\circ} \mathrm{C}$ (Aspergillus) or at $30^{\circ} \mathrm{C}$ (Fusarium and Alternaria), and the number of $\mathrm{CFU} /$ eye was determined by direct counting. The weight of the whole eye is consistent from one mouse to the next regardless of infection, and as we homogenize the entire eye and not just the cornea, we calculate CFU from the entire eye not just a representative sample. Fungal dsRed and CFU analysis do not have a linear correlation as hyphae of varying lengths show differences in dsRed fluorescence, but are still counted as a single CFU. Also, as homogenization can potentially damage branched hyphae, which may be more abundant in wild type compared with mutants, we may be underestimating the difference in CFUs between strains. All CFU graphs show pooled data from at least three repeat experiments.

\section{Quantification of IL-6 protein in mouse corneas and serum}

Corneas were homogenized in $150 \mu \mathrm{l}$ reagent diluent $(\mathrm{R} \& \mathrm{D}$ Systems, Minneapolis, MN) using the Retsch MM 300 ball miller at $33 \mathrm{~Hz}$ for $4 \mathrm{~min}$ (Qiagen). Mouse serum was obtained as described below and assayed directly. IL-6 protein was quantified using a mouse IL-6 ELISA kit as per manufacturer's instructions (R \& D Systems, Minneapolis, MN).

\section{Quantification of neutrophils in mouse corneas}

Corneas were dissected, cut into 8 small fragments, and incubated in 80 units of collaganese (Sigma-Aldrich) for 1-2 h. The cell suspensions were filtered, centrifuged at $300 * \mathrm{~g}$ for $5 \mathrm{~min}$ at $4{ }^{\circ} \mathrm{C}$ and washed in FACS buffer (PBS+1\% $\mathrm{FBS}+0.5 \% \mathrm{Na}$ azide). Cells were then incubated with anti-mouse CD16/32 antibody (Fc block, clone 93, eBioscience) for $10 \mathrm{~min}$ followed immediately by incubation with biotinylated rat anti-mouse NIMP-R14 or isotype-control for $45 \mathrm{~min}$. Cells were washed and incubated with streptavidin-PE-Cy7 for $30 \mathrm{~min}$ in the dark. Cell suspensions were then analyzed utilizing a C6 Accuri flow cytometer with gates set based on isotype controls.

\section{Quantitative PCR of infected corneas}

C57BL/6 mice and IL- $6^{-/-}$mice were infected with $A$. fumigatus strain Af-dsRed as described above. At $24 \mathrm{~h}$ mice were sacrificed, corneas were excised, suspended in tissue lysis buffer (Qiagen, Valencia, CA) and homogenized using the Mixer Mill MM300 (Retsch) at $33 \mathrm{~Hz}$ for 2 min. Subsequently, RNA was extracted from samples using RNeasy mini kit according to the manufacturer's directions (Qiagen, Valencia, CA). Samples with a 260/280 (RNA:protein) ratio of 2.0 were used to generate cDNA using the superscript first strand synthesis system (Life technologies, Grand Island, NY) using standard methods. Real Time PCR was performed on the cDNA samples using the SYBR green 
Table 1. Fungal strains utilized in this study.

\begin{tabular}{|c|c|c|}
\hline Strain & Genotype & Phenotype \\
\hline \multicolumn{3}{|l|}{ A. fumigatus } \\
\hline Af-dsRed [23] & Af293.1- $\Delta$ pyrG1::gpdA::dsRed::pyrG & dsRed Fluorescence \\
\hline ATCC 46645 & & WT \\
\hline$\Delta$ sidA [29] & ATCC 46645- sidA::hph & No intra or extracellular siderophores \\
\hline $\operatorname{sid} A^{R}[29]$ & $\Delta$ sidA- sidA::sidA & Complemented strain \\
\hline$\Delta \operatorname{sidC}[11]$ & ATCC 46645- sidC::hph & No intracellular siderophores \\
\hline$\Delta s i d D[11]$ & ATCC 46645- sidD::hph & No fusarinine $C$ or TAFC \\
\hline $\operatorname{sid} D^{R}[11]$ & $\Delta$ sidD- sidD::sidD & Complemented strain \\
\hline$\Delta \operatorname{sid} F[11]$ & ATCC 46645- sidF::hph & No extracellular siderophores \\
\hline $\operatorname{sidF}^{R}[11]$ & $\Delta$ sidF- sidF:: sidF & Complemented strain \\
\hline$\Delta s i d G[11]$ & ATCC 46645- sidG::hph & No TAFC \\
\hline$\Delta s i d H[14]$ & ATCC 46645- sidH::hph & No extracellular siderophores \\
\hline $\operatorname{sid} H^{R}[14]$ & $\Delta$ sidH- sidH:::sidH & Complemented strain \\
\hline$\Delta \operatorname{sidl}[14]$ & ATCC 46645- sidl::hph & No extracellular siderophores \\
\hline $\operatorname{sid}^{R}[14]$ & $\Delta$ sidl- sidl::sidl & Complemented strain \\
\hline$\Delta$ hapX [30] & ATCC 46645- hapX::hph & No hapX \\
\hline $\operatorname{hap} X^{R}[30]$ & $\Delta$ hapX- hapX::hapX & Complemented strain \\
\hline$\Delta f t r A[29]$ & ATCC 46645- ftrA::hph & No reductive iron assimilation \\
\hline \multicolumn{3}{|l|}{ F. oxysporum } \\
\hline FoxL- 4287 [37] & & WT \\
\hline$\Delta$ hapX [37] & 4287- hapX::hph & No hapX \\
\hline $\operatorname{hap} X^{R}[37]$ & $\Delta$ hapX- hapX::hapX & Complemented strain \\
\hline \multicolumn{3}{|l|}{ A. brassicicola } \\
\hline WT-Tf383 & & WT \\
\hline$\Delta n p s 2[38]$ & Tf383- nps2::hph & No intracellular siderophores \\
\hline$\Delta n p s 6[38]$ & Tf383- nps6::hph & No extracellular siderophores \\
\hline$\Delta n p s 2 / 6[38]$ & Tf383- nps2::hph; nps6::hph & No intra or extracellular siderophores \\
\hline
\end{tabular}

system (Applied Biosystems, Carlsbad, CA). All primers used in this study are listed in Table 2 and were synthesized by Integrated DNA technologies (San Diego, CA). Fold change with respect to naive uninfected corneas was calculated using the $2^{-\Delta \Delta \mathrm{ct}}$ method. Data are therefore presented as fold increases of relative gene expression $(\log (\mathrm{RQ})$ ). RT-PCR samples were also analyzed by $2 \%$ agarose gel electrophoresis.

\section{Quantification of iron content in mouse serum}

Whole blood was obtained from mice by retro-orbital bleeding, and serum was recovered following blood coagulation. An iron assay kit (ABCAM, Cambridge, MA) was subsequently used to quantify $\mathrm{Fe}^{2+}$ and $\mathrm{Fe}^{3+}$ in the serum using manufacturer's instructions. Briefly, $25 \mu \mathrm{l}$ of serum was added to $75 \mu \mathrm{l}$ iron assay buffer and $5 \mu$ iron reducer, which reduces $\mathrm{Fe}^{3+}$ to $\mathrm{Fe}^{2+}$. Next, $100 \mu \mathrm{l}$ of the iron-probe solution was added yielding a $\mathrm{Fe}^{2+}$-ferene $\mathrm{S}$ complex that absorbs light at $593 \mathrm{~nm}$. Spectrophotometry was used to detect absorbance at this wavelength.

\section{In vitro human neutrophil:hyphae growth inhibition assay}

Human neutrophils were isolated from normal, healthy donors using Ficoll-Paque Plus (GE) density centrifugation as described [8]. Isolated conidia from each $A$. fumigatus mutant were cultured in
$200 \mu \mathrm{l}$ SDA media (12,500/well) in black-wall 96 well plates with an optically clear bottom (CoStar 3720) until early germ tubes were observed $(4-6 \mathrm{~h})$. Wells were washed twice with sterile $\mathrm{ddH}_{2} \mathrm{O}$ and incubated $16 \mathrm{~h}$ with either RPMI media (+ Control), PBS (Control), or human peripheral blood neutrophils suspended in RPMI at $0.5-1 * 10^{5} /$ well, which we know does not inhibit fungal growth [8]. After $16 \mathrm{~h}$ incubation, plates were washed and stained with $50 \mu \mathrm{l}$ calcofluor white stain, which binds chitin (Fluka 18909) for $5 \mathrm{~min}$ in the dark. Subsequently, plates were washed three times with $\mathrm{dd}_{2} \mathrm{O}$ and quantified by fluorometry (360/440 nm; Synergy HT; Biotek). In assays where no neutrophils were added, fungi were incubated in SDA media alone with or without inhibitors. Fungi cultured in 96well plates exhibit maximal growth by $16 \mathrm{~h}$; therefore, this assay measures only relative decreases in fungal growth.

\section{Statistical analysis}

Statistical analysis was performed for each experiment using one way ANOVA with a Tukey post-hoc analysis using Prism software (GraphPad Software Inc, La Jolla, CA). A p value $<0.05$ was considered significant.

\section{Ethics statement}

All animals were treated in accordance with the guidelines provided in the Association for Research in Vision and Ophthal- 
Table 2. Primers sequences and protein function.

\begin{tabular}{|c|c|c|c|}
\hline Primer & Source (PB ID) & Sequence & Protein Function \\
\hline \multicolumn{4}{|l|}{ Iron Chelation } \\
\hline \multirow[t]{2}{*}{ Lactoferrin } & $141803548 \mathrm{~b} 2$ & CCGCTCAGTTGTGTCAAGAAA & Binds $\mathrm{Fe}$ at low $\mathrm{pH}$ \\
\hline & & CATGGCATCAGCTCTGTTTGT & \\
\hline \multirow[t]{2}{*}{ Transferrin } & $118129942 \mathrm{~b} 1$ & GCTGTCCCTGACAAAACGGT & Binds Fe at neutral $\mathrm{pH}$ \\
\hline & & GGTATTCTCGTGCTCTGACAC & \\
\hline \multirow[t]{2}{*}{ Tf receptor 1} & 291045184b1 & GATCAAGCCAGATCAGCATTCT & Internalizes transferrin \\
\hline & & GTGTATGACAATGGTTCCCCAC & \\
\hline \multirow[t]{2}{*}{ Tf receptor 2} & $113204629 \mathrm{~b} 1$ & CGTTGGGGTCTACTTCGGAGA & Internalizes transferrin \\
\hline & & AGATGGTCTGAGAGGGTCTTG & \\
\hline \multirow[t]{2}{*}{ Lf receptor/intelectin 1} & $118130045 \mathrm{~b} 1$ & CAGCACTTGGGACATAATCTGT & Internalizes lactoferrin \\
\hline & & TCCTTCTCCGTATTTCACTGGG & \\
\hline \multirow[t]{2}{*}{ Lipocalin-1- Human } & 32455234b1 & ATGTGTCAGGGACGTGGTATC & Binds fungal siderophores \\
\hline & & CCGATTCCAGATTCATCTCAGG & \\
\hline \multicolumn{4}{|l|}{ Heme Binding Protein } \\
\hline \multirow[t]{2}{*}{ Haptoglobin } & $254910958 \mathrm{~b} 1$ & GCACTTGGTTCGCTATCGCT & Binds hemoglobin \\
\hline & & GCCCGTAGTCTGTAGAACTGT & \\
\hline \multirow[t]{2}{*}{ Hemopexin } & $160358828 b 1$ & AGCAGTGGCGCTAAATATCCT & Binds heme \\
\hline & & CAACTCTCCCGTTGGCAGTA & \\
\hline \multicolumn{4}{|l|}{ Siderophore Binding } \\
\hline \multirow[t]{2}{*}{ Lipocalin-1 } & $32455234 b$ & ATGTGTCAGGGACGTGGTATC & Binds fungal siderophores \\
\hline & & CCGATTCCAGATTCATCTCAGG & \\
\hline \multirow[t]{2}{*}{ Lipocalin-2 } & 34328048b1 & GCAGGTGGTACGTTGTGGG & Binds bacterial siderophores \\
\hline & & CTCTTGTAGCTCATAGATGGTGC & \\
\hline \multicolumn{4}{|l|}{ Hepcidin Signaling } \\
\hline \multirow[t]{2}{*}{ Interleukin 6} & 13624310b1 & CTGCAAGAGACTTCCATCCAG & Induces HAMP* synthesis \\
\hline & & AGTGGTATAGACAGGTCTGTTGG & \\
\hline \multirow[t]{2}{*}{ HFE } & 145966687b1 & CGGGCTGCCTTTGTTTGAG & Signal transduction $\rightarrow$ HAMP \\
\hline & & CTGGCTTGAGGTTTGCTCC & \\
\hline \multirow[t]{2}{*}{ BMP-2 } & 71896668b1 & TCTTCCGGGAACAGATACAGG & Amplifies HAMP synthesis \\
\hline & & TGGTGTCCAATAGTCTGGTCA & \\
\hline \multirow[t]{2}{*}{ BMP-4 } & $121949822 \mathrm{~b} 1$ & ATTCCTGGTAACCGAATGCTG & “ \\
\hline & & CCGGTCTCAGGTATCAAACTAGC & \\
\hline \multirow[t]{2}{*}{ BMP6 } & 118131176b1 & GCGGGAGATGCAAAAGGAGAT & “ \\
\hline & & ATTGGACAGGGCGTTGTAGAG & \\
\hline \multirow[t]{2}{*}{ alk1- BMPR type 1a } & 133891829b1 & TGGCACTGGTATGAAATCAGAC & $\mathrm{BMP}^{*}$ receptor \\
\hline & & CAAGGTATCCTCTGGTGCTAAAG & \\
\hline \multirow[t]{2}{*}{ alk2-BMPR type $1 \mathrm{~b}$} & $41053831 \mathrm{~b} 1$ & CCTCGGCCCAAGATCCTAC & $"$ \\
\hline & & CCTAGACATCCAGAGGTGACA & \\
\hline \multirow[t]{2}{*}{ actrllA-BMPR type 2} & 145966831b1 & GTGTTATGGTCTGTGGGAGAAAT & “ \\
\hline & & AAAGCGGTACGTTCCATTCTG & \\
\hline Hemojuvelin & 166295197b1 & ATGGGCCAGTCCCCTAGTC & BMPR signaling \\
\hline & & CAGCGGAGGATCTTGCACT & \\
\hline Hepcidin & Blood 2010 [63] & CTGAGCAGCACCACCTATCTC & Degrades ferroportin \\
\hline & & TGGCTCTAGGCTATGTTTTGC & \\
\hline Ferroportin & $124248584 \mathrm{~b} 1$ & TGGAACTCTATGGAAACAGCCT & Exports iron from cells \\
\hline & & TGGCATTCTTATCCACCCAGT & \\
\hline$\underline{\text { Housekeeping gene }}$ & & & \\
\hline$\beta$-actin- Mouse & $6671509 a 1$ & GGCTGTATTCCCCTCCATCG & Housekeeping gene \\
\hline & & CCAGTTGGTAACAATGCCATGT & \\
\hline
\end{tabular}


Table 2. Cont.

\begin{tabular}{llll}
\hline & & \\
\hline Primer & Source (PB ID) & Sequence & Protein Function \\
\hline$\beta$-actin - Human & 144922730c1 & GTCTGCCTTGGTAGTGGATAATG & Housekeeping gene \\
& & TCGAGGACGCCCTATCATGG & \\
\hline
\end{tabular}

*BMP: bone morphogenetic protein, HAMP: hepcidin, HFE: human hemochromatosis protein, Tf: transferrin.

doi:10.1371/journal.ppat.1003436.t002

mology ARVO statement for the Use of Animals in Ophthalmic and Vision Research, and were approved by Case Western Reserve University IACUC (2011-0063). The protocol for the use of human peripheral blood from normal healthy volunteers was approved by the Institutional Review Board at University Hospitals of Cleveland (07-11-21). Informed consent was obtained in writing from each volunteer.

\section{Supporting Information}

Figure $\mathrm{S} 1$ Complementation of $A$. fumigatus and $F$. oxysporum mutant strains causes phenotypic reversion to WT parental strain phenotypes. A. C57BL/6 mice were infected with 40,000 conidia from the WT A. fumigatus strain ATCC 46645 and the the complemented strains: $\operatorname{sid}^{\mathrm{R}}$, $\operatorname{sidD}^{\mathrm{R}}$, $\operatorname{sid}^{\mathrm{R}}, \operatorname{sid} \mathrm{H}^{\mathrm{R}}$, sidI $\mathrm{I}^{\mathrm{R}}$, hapX $\mathrm{X}^{\mathrm{R}}$ and $\mathrm{CFU}$ analysis was performed at $48 \mathrm{~h}$ post-infection. B. C57BL/6 mice were infected with 30,000 conidia from the WT $F$. oxysporum strain 4287 and the complemented strain hap $\mathrm{X}^{\mathrm{R}}$ and CFU analysis was performed at $48 \mathrm{~h}$ post-infection. C. A. fumigatus and D. F. oxysporum infected eyes were imaged at $24 \mathrm{~h}$ and $48 \mathrm{~h}$ post-infection.

(TIFF)

\section{References}

1. Milner JD, Sandler NG, Douek DC (2010) Th17 cells, Job's syndrome and HIV: opportunities for bacterial and fungal infections. Curr Opin HIV AIDS 5: 179-183.

2. Antachopoulos C (2010) Invasive fungal infections in congenital immunodeficiencies. Clin Microbiol Infect 16: 1335-1342.

3. Thomas PA (2003) Fungal infections of the cornea. Eye 17: 852-862.

4. Gower EW, Keay LJ, Oechsler RA, Iovieno A, Alfonso EC, et al. (2010) Trends in fungal keratitis in the United States, 2001 to 2007. Ophthalmology 117: 2263-2267.

5. World Health Organization (October 2011) Visual Impairment and BlindnessKey Facts. Centre WM, editor.

6. Whitcher JP, Srinivasan M, Upadhyay MP (2001) Corneal blindness: a global perspective. Bull World Health Organ 79: 214-221.

7. London Fungal Research Trust (2011) How Common are fungal diseases? Available: www.fungalinfectiontrust.org/HowCommonareFungalDiseases2.pdf.

8. Leal SM, Jr., Vareechon C, Cowden S, Cobb BA, Latge JP, et al. (2012) Fungal antioxidant pathways promote survival against neutrophils during infection. J Clin Invest 122: 2482-2498.

9. Wood ZA, Schroder E, Robin Harris J, Poole LB (2003) Structure, mechanism and regulation of peroxiredoxins. Trends Biochem Sci 28: 32-40.

10. Winterbourn CG (2008) Reconciling the chemistry and biology of reactive oxygen species. Nat Chem Biol 4: 278-286.

11. Schrettl M, Bignell E, Kragl C, Sabiha Y, Loss O, et al. (2007) Distinct roles for intra- and extracellular siderophores during Aspergillus fumigatus infection. PLoS Pathog 3: 1195-1207.

12. Haas H, Eisendle M, Turgeon BG (2008) Siderophores in fungal physiology and virulence. Annu Rev Phytopathol 46: 149-187.

13. Haas H (2012) Iron - A Key Nexus in the Virulence of Aspergillus fumigatus. Front Microbiol 3: 28.

14. Yasmin S, Alcazar-Fuoli L, Grundlinger M, Puempel T, Cairns T, et al. (2012) Mevalonate governs interdependency of ergosterol and siderophore biosyntheses in the fungal pathogen Aspergillus fumigatus. Proc Natl Acad Sci U S A 109: E497-504.

15. Hentze MW, Muckenthaler MU, Galy B, Camaschella C (2010) Two to tango: regulation of Mammalian iron metabolism. Cell 142: 24-38.

16. Flanagan JL, Willcox MD (2009) Role of lactoferrin in the tear film. Biochimie 91: $35-43$.

17. Fluckinger M, Haas H, Merschak P, Glasgow BJ, Redl B (2004) Human tear lipocalin exhibits antimicrobial activity by scavenging microbial siderophores. Antimicrob Agents Chemother 48: 3367-3372.
Figure S2 Siderophores are required for survival of Alternaria during infection. A. C57BL/6 mice were infected with 40,000 conidia from the Alternaria brassicicola strain Tf383, and the isogenic mutant strains: $\Delta \mathrm{nps} 2$ (no intracellular siderophores), $\Delta$ nps6 (no extracellular siderophores), and $\Delta$ nps2/6 (no intracellular or extracellular siderophores) and CFU analysis was performed at $48 \mathrm{~h}$ post-infection. B. Eyes were imaged at $24 \mathrm{~h}$ and $48 \mathrm{~h}$ post-infection $\mathbf{C}$. Metamorph image analysis was used to quantify both cornea opacity area and $\mathbf{D}$. total cornea opacity. (TIFF)

\section{Acknowledgments}

We would like to thank Dr. B. Gillian Turgeon for helpful discussions and the CWRU visual sciences core facility managers for outstanding technical assistance.

\section{Author Contributions}

Conceived and designed the experiments: SML EP HH. Performed the experiments: SML SR SdC CV HC. Analyzed the data: SML EP HH. Contributed reagents/materials/analysis tools: MSLB MS HH BR NB AdP. Wrote the paper: SML EP.

18. Skaar EP (2010) The battle for iron between bacterial pathogens and their vertebrate hosts. PLoS Pathog 6: e1000949.

19. Amulic B, Cazalet C, Hayes GL, Metzler KD, Zychlinsky A (2012) Neutrophil function: from mechanisms to disease. Annu Rev Immunol 30: 459-489.

20. Armitage AE, Eddowes LA, Gileadi U, Cole S, Spottiswoode N, et al. (2011) Hepcidin regulation by innate immune and infectious stimuli. Blood 118: 4129-4139.

21. Peyssonnaux G, Zinkernagel AS, Datta V, Lauth X, Johnson RS, et al. (2006) TLR4-dependent hepcidin expression by myeloid cells in response to bacterial pathogens. Blood 107: 3727-3732.

22. Ganz T, Nemeth E (2011) Hepcidin and disorders of iron metabolism. Annu Rev Med 62: 347-360.

23. Leal SM, Jr., Cowden S, Hsia YC, Ghannoum MA, Momany M, et al. (2010) Distinct roles for Dectin-1 and TLR4 in the pathogenesis of Aspergillus fumigatus keratitis. PLoS Pathog 6: e1000976.

24. Karthikeyan RS, Leal SM, Jr., Prajna NV, Dharmalingam K, Geiser DM, et al. (2011) Expression of innate and adaptive immune mediators in human corneal tissue infected with Aspergillus or fusarium. J Infect Dis 204: 942-950.

25. Hider RC, Kong X (2010) Chemistry and biology of siderophores. Nat Prod Rep 27: 637-657.

26. Correnti C, Strong RK (2012) Mammalian siderophores, siderophore-binding lipocalins, and the labile iron pool. J Biol Chem 287: 13524-13531.

27. Dartt DA (2011) Tear lipocalin: structure and function. Ocul Surf 9: 126-138.

28. Petrik M, Haas H, Schrettl M, Helbok A, Blatzer M, et al. (2012) In vitro and in vivo evaluation of selected 68Ga-siderophores for infection imaging. Nucl Med Biol 39: 361-369.

29. Schrettl M, Bignell E, Kragl C, Joechl C, Rogers T, et al. (2004) Siderophore biosynthesis but not reductive iron assimilation is essential for Aspergillus fumigatus virulence. J Exp Med 200: 1213-1219.

30. Schrettl M, Beckmann N, Varga J, Heinekamp T, Jacobsen ID, et al. (2010) HapX-mediated adaption to iron starvation is crucial for virulence of Aspergillus fumigatus. PLoS Pathog 6: e1001124.

31. Flo TH, Smith KD, Sato S, Rodriguez DJ, Holmes MA, et al. (2004) Lipocalin 2 mediates an innate immune response to bacterial infection by sequestrating iron. Nature 432: 917-921.

32. Raffatellu M, George MD, Akiyama Y, Hornsby MJ, Nuccio SP, et al. (2009) Lipocalin-2 resistance confers an advantage to Salmonella enterica serotype Typhimurium for growth and survival in the inflamed intestine. Cell Host Microbe 5: 476-486. 
33. Corsini A, Bellosta S, Baetta R, Fumagalli R, Paoletti R, et al. (1999) New insights into the pharmacodynamic and pharmacokinetic properties of statins. Pharmacol Ther 84: 413-428.

34. Zamocky M, Koller F (1999) Understanding the structure and function of catalases: clues from molecular evolution and in vitro mutagenesis. Prog Biophys Mol Biol 72: 19-66.

35. Hohl TM, Van Epps HL, Rivera A, Morgan LA, Chen PL, et al. (2005) Aspergillus fumigatus triggers inflammatory responses by stage-specific betaglucan display. PLoS Pathog 1: e30.

36. Gersuk GM, Underhill DM, Zhu L, Marr KA (2006) Dectin-1 and TLRs permit macrophages to distinguish between different Aspergillus fumigatus cellular states. J Immunol 176: 3717-3724.

37. Manuel Berges JC, David Turra, Lukas Schafferer, Sandra Matthijs, Christoph Jochl, Pierre Cornelis, Josep Guarro, Hubertus Haas, Antonio Di Pietro (2012) HapX-Mediated Iron Homeostasis Is Essential for Rhizosphere Competence and Virulence of the Soilborne Pathogen Fusarium oxysporum. Plant Cell 24: 3805-3822.

38. Oide S, Moeder W, Krasnoff S, Gibson D, Haas H, et al. (2006) NPS6, encoding a nonribosomal peptide synthetase involved in siderophore-mediated iron metabolism, is a conserved virulence determinant of plant pathogenic ascomycetes. Plant Cell 18: 2836-2853.

39. Liu H, Gravelat FN, Chiang LY, Chen D, Vanier G, et al. (2010) Aspergillus fumigatus AcuM regulates both iron acquisition and gluconeogenesis. Mol Microbiol 78: 1038-1054.

40. Smith MEB, Lee NJ, Haney E, Carson S (2009) Drug Class Review: HMG-CoA Reductase Inhibitors (Statins) and Fixed-dose Combination Products Containing a Statin: Final Report Update 5. In: University OHS, editor. Drug Class Review: HMG-CoA Reductase Inhibitors (Statins) and Fixed-dose Combination Products Containing a Statin: Final Report Update 5. Portland (OR).

41. Macreadie IG, Johnson G, Schlosser T, Macreadie PI (2006) Growth inhibition of Candida species and Aspergillus fumigatus by statins. FEMS Microbiol Lett 262: 9-13.

42. Qiao J, Kontoyiannis DP, Wan Z, Li R, Liu W (2007) Antifungal activity of statins against Aspergillus species. Med Mycol 45: 589-593.

43. Nyilasi I, Kocsube S, Krizsan K, Galgoczy L, Pesti M, et al. (2010) In vitro synergistic interactions of the effects of various statins and azoles against some clinically important fungi. FEMS Microbiol Lett 307: 175-184.

44. Wojnar P, Lechner M, Redl B (2003) Antisense down-regulation of lipocalininteracting membrane receptor expression inhibits cellular internalization of lipocalin-1 in human NT2 cells. J Biol Chem 278: 16209-16215.

45. Wojnar P, Lechner M, Merschak P, Redl B (2001) Molecular cloning of a novel lipocalin-1 interacting human cell membrane receptor using phage display. J Biol Chem 276: 20206-20212.

46. Fullard RJ, Kissner DM (1991) Purification of the isoforms of tear specific prealbumin. Curr Eye Res 10: 613-628.

47. Zarember KA, Sugui JA, Chang YC, Kwon-Chung KJ, Gallin JI (2007) Human polymorphonuclear leukocytes inhibit Aspergillus fumigatus conidial growth by lactoferrin-mediated iron depletion. J Immunol 178: 6367-6373.
48. Ma Y, Zhou T, Kong X, Hider RC (2012) Chelating agents for the treatment of systemic iron overload. Curr Med Chem 19: 2816-2827.

49. Robins-Browne RM, Prpic JK (1985) Effects of iron and desferrioxamine on infections with Yersinia enterocolitica. Infect Immun 47: 774-779.

50. Boelaert JR, van Roost GF, Vergauwe PL, Verbanck JJ, de Vroey C, et al. (1988) The role of desferrioxamine in dialysis-associated mucormycosis: report of three cases and review of the literature. Clin Nephrol 29: 261-266.

51. Windus DW, Stokes TJ, Julian BA, Fenves AZ (1987) Fatal Rhizopus infections in hemodialysis patients receiving deferoxamine. Ann Intern Med 107: 678-680.

52. Christopher GW (1985) Escherichia coli bacteremia, meningitis, and hemochromatosis. Arch Intern Med 145: 1908.

53. Bullen JJ, Spalding PB, Ward CG, Gutteridge JM (1991) Hemochromatosis, iron and septicemia caused by Vibrio vulnificus. Arch Intern Med 151: 1606-1609.

54. Abbott M, Galloway A, Cunningham JL (1986) Haemochromatosis presenting with a double Yersinia infection. J Infect 13: 143-145.

55. Kontoyiannis DP, Chamilos G, Lewis RE, Giralt S, Cortes J, et al. (2007) Increased bone marrow iron stores is an independent risk factor for invasive aspergillosis in patients with high-risk hematologic malignancies and recipients of allogeneic hematopoietic stem cell transplantation. Cancer 110: 1303-1306.

56. Altes A, Remacha AF, Sarda P, Sancho FJ, Sureda A, et al. (2004) Frequent severe liver iron overload after stem cell transplantation and its possible association with invasive aspergillosis. Bone Marrow Transplant 34: 505-509.

57. Zarember KA, Cruz AR, Huang CY, Gallin JI (2009) Antifungal activities of natural and synthetic iron chelators alone and in combination with azole and polyene antibiotics against Aspergillus fumigatus. Antimicrob Agents Chemother 53: 2654-2656.

58. Ibrahim AS, Gebremariam T, French SW, Edwards JE, Jr., Spellberg B (2010 The iron chelator deferasirox enhances liposomal amphotericin B efficacy in treating murine invasive pulmonary aspergillosis. J Antimicrob Chemother 65: 289-292.

59. Ibrahim AS, Gebermariam T, Fu Y, Lin L, Husseiny MI, et al. (2007) The iron chelator deferasirox protects mice from mucormycosis through iron starvation. J Clin Invest 117: 2649-2657.

60. Ibrahim AS, Edwards JE, Jr., Fu Y, Spellberg B (2006) Deferiprone iron chelation as a novel therapy for experimental mucormycosis. J Antimicrob Chemother 58: 1070-1073.

61. Spellberg B, Ibrahim AS, Chin-Hong PV, Kontoyiannis DP, Morris MI, et al. (2012) The Deferasirox-AmBisome Therapy for Mucormycosis (DEFEAT Mucor) study: a randomized, double-blinded, placebo-controlled trial. J Antimicrob Chemother 67: 715-722.

62. Kontoghiorghes GJ (2007) Deferasirox: uncertain future following renal failure fatalities, agranulocytosis and other toxicities. Expert Opin Drug Saf 6: 235-239.

63. Gao J, Chen J, De Domenico I, Koeller DM, Harding CO, et al. (2010) Hepatocyte-targeted HFE and TFR2 control hepcidin expression in mice. Blood 115: 3374-3381. 